La Revue

des Droits

de l'Homme

\section{La Revue des droits de l'homme}

Revue du Centre de recherches et d'études sur les droits fondamentaux

Actualités Droits-Libertés | 2015

Cour européenne des droits de l'homme : De l'art de la résilience juridictionnelle

Cour européenne des droits de l'homme (Bilan annuel)

\title{
Nicolas Hervieu
}

\section{OpenEdition}

Journals

Édition électronique

URL : https://journals.openedition.org/revdh/1062

DOI : $10.4000 /$ revdh. 1062

ISSN : 2264-119X

Éditeur

Centre de recherches et d'études sur les droits fondamentaux

Référence électronique

Nicolas Hervieu, «Cour européenne des droits de l'homme : De l'art de la résilience juridictionnelle », La Revue des droits de l'homme [En ligne], Actualités Droits-Libertés, mis en ligne le 16 février 2015 ,

consulté le 24 janvier 2022. URL : http://journals.openedition.org/revdh/1062 ; DOI : https://doi.org/ 10.4000/revdh.1062

Ce document a été généré automatiquement le 24 janvier 2022.

Tous droits réservés 


\title{
Cour européenne des droits de l'homme : De l'art de la résilience juridictionnelle
}

Cour européenne des droits de l'homme (Bilan annuel)

\author{
Nicolas Hervieu
}

Evoluer pour s'adapter et survivre dans un environnement hostile. Mais par hérédité, conserver ses traits les plus déterminants. Le tout, afin de maintenir intacte sa vocation originelle de "sauvegarde et de développement des droits de l'homme" inscrite au frontispice de son acte fondateur. Ainsi décrite, la Cour européenne des droits de l'homme pourrait aisément être qualifiée de juridiction néo-darwiniste. D'aucuns jugeront l'épithète bien aventureux. Pourtant, à l'orée de l'année 2015 et à l'heure de dresser le bilan de l'année écoulée, un simple regard sur le chemin juridictionnel parcouru donne corps à cette vision. Car malgré quelques errements et erreurs, force est de constater que la Cour de Strasbourg a fait preuve de résilience juridictionnelle devant l'adversité. Plus significatif encore, même «les crises temporaires et controverses » subies n'ont pas été "des signes de défaites mais plutôt la preuve d'une évolution, conformément (...) à l'idée d'éclatements et d'équilibres ponctuels évoqués dans la théorie néodarwiniste de l'évolution $»^{1}$.

Certes, résilience n'est aucunement synonyme de perfection. Régulièrement, tant le fonctionnement de la Cour que la teneur de sa jurisprudence prêtent le flanc à la critique. Mais être perpétuellement poursuivie par la vindicte des contempteurs est inévitable pour une juridiction si souvent saisie de "questions très sensibles" et constamment appelée à prendre "position dans des matières infiniment complexes $»^{2}$. Les tensions qui en résultent ne sont toutefois pas insurmontables. Pour les dissoudre avec diplomatie, chacun comprendra que la Cour préfère nier toute "stratégie juridictionnelle $\|^{3}$ pour s'afficher en simple organisateur de la " polyrythmie », trait que le système européen des droits de l'homme aurait en partage avec la Danse sacrale de Stravinski $\mathrm{i}^{4}$. Mais pour poursuivre la métaphore musicale chère au Président Dean Spielmann, la partition de la Convention européenne tient aussi du Concerto pour 
violon de Tchaïkovski : une œuvre qui n'est rien sans l'effort collectif de l'orchestre, mais qui ne prend son sens que si le soliste strasbourgeois parvient à l'exécuter en dépit de son extrême difficulté. Quitte, pour ce faire, à donner fermement le diapason à l'ensemble des acteurs étatiques.

Inévitablement, dans ce contexte tendu, il n'est pas rare que quelques fausses notes retentissent. Mais ces dissonances doivent être réduites à leur juste mesure. Sans minimisation, ni extrapolation. Surtout, il importe de les replacer dans une temporalité plus longue, les coups d'éclats ponctuels ne devant pas occulter pas des tendances pérennes. D'où la nécessité de conjuguer le bilan annuel de la Cour à tous les temps ${ }^{5}$. Au passé, d'abord, car l'inventaire de l'année écoulée confirme l'encourageante embellie statistique née des efforts européens $\left(1^{\circ}\right)$. Au présent, ensuite, puisqu'aujourd'hui le centre des gravité des droits de l'homme en Europe oscille fortement sous les coups habituels - de certains acteurs étatiques et ceux - plus inattendus - venus de Luxembourg $\left(2^{\circ}\right)$. Au futur, enfin, car l'écriture d'un nouveau chapitre européen s'annonce des plus riches, à l'approche d'un important renouvellement de la Cour et de nouvelles échéances institutionnelles $\left(3^{\circ}\right)$.

\section{$1 \%$ - Un passé encourageant : La fructueuse récolte des efforts européens}

4 Maîtriser les flots contentieux qui déferlent sur la Cour européenne. Pendant longtemps, ce seul objectif relevait en soi de la gageure, tant la situation était critique à Strasbourg. Pourtant, aux premières heures de l'année 2015, telle est bien la perspective qui se profile résolument. Dès lors, sous le prisme quantitatif, le bilan de l'année écoulée ne peut que réjouir (A). Mais cette seule lecture ne saurait suffire et doit être complétée par un regard qualitatif dont l'issue est moins positive (B).

\section{A - Le prisme quantitatif : Une salutaire «tendance à la décroissance » contentieuse}

5 Spectaculaire, l'embellie statistique au cours de l'année 2015 était cependant prévisible (1), même si elle n'en est pas moins salutaire (2).

\section{1) Un reflux contentieux prévisible}

6 De prime abord, l'autosatisfaction pourrait sembler être une constante à Strasbourg. En effet, ces dernières années la Cour européenne et ses plus hauts représentants n'ont cessé de verser dans le triomphalisme à l'heure de présenter les résultats statistiques obtenus. Mais comme l'an passé, ce réflexe européen s'explique par l'immense soulagement d'une institution en passe de surmonter une menace mortelle ${ }^{6}$ : l'engorgement de son prétoire par un flot de requêtes.

7 A cet égard, il importe de rappeler qu'au cours des années 2000, la Cour a subi de plein fouet un accroissement exponentiel du nombre de requêtes, passé de 10000 à près de 65000 en 2011. Ce mouvement a eu pour conséquence d'élever l'arriéré de requêtes pendantes - également désigné à Strasbourg sous le nom de "back log " - jusqu'au pic alarmant de 151600 requêtes en 2011. Mais dès l'année 2012, une véritable décrue de 
cet arriéré s'est amorcée. Ainsi, ce chiffre est passé à 128000 en $2012^{7}$ jusqu'à basculer en 2013 en dessous de la barre symbolique des 100000 requêtes $^{8}$.

8 Certes, avec toujours 800 millions de justiciables potentiels en Europe, la Cour n'a guère pu compter sur une réduction du nombre de nouvelles requêtes, lequel est resté relativement stable en 2012 et $2013^{9}$. Mais à la faveur d'une augmentation de la productivité, ce nombre de requêtes "entrantes» a été nettement dépassé par le nombre d'affaires "sortantes", réduisant mécaniquement le stock de requêtes en souffrance.

9 En 2014, et sans aucune surprise, cette "tendance à la décroissance » ${ }^{10}$ s'est poursuivie. Ainsi, comme l'illustre le spectaculaire graphique des requêtes pendantes, le nombre de requêtes pendantes a été réduit de $30 \%$, passant de 99900 en 2013 à 69900 en 2014 :

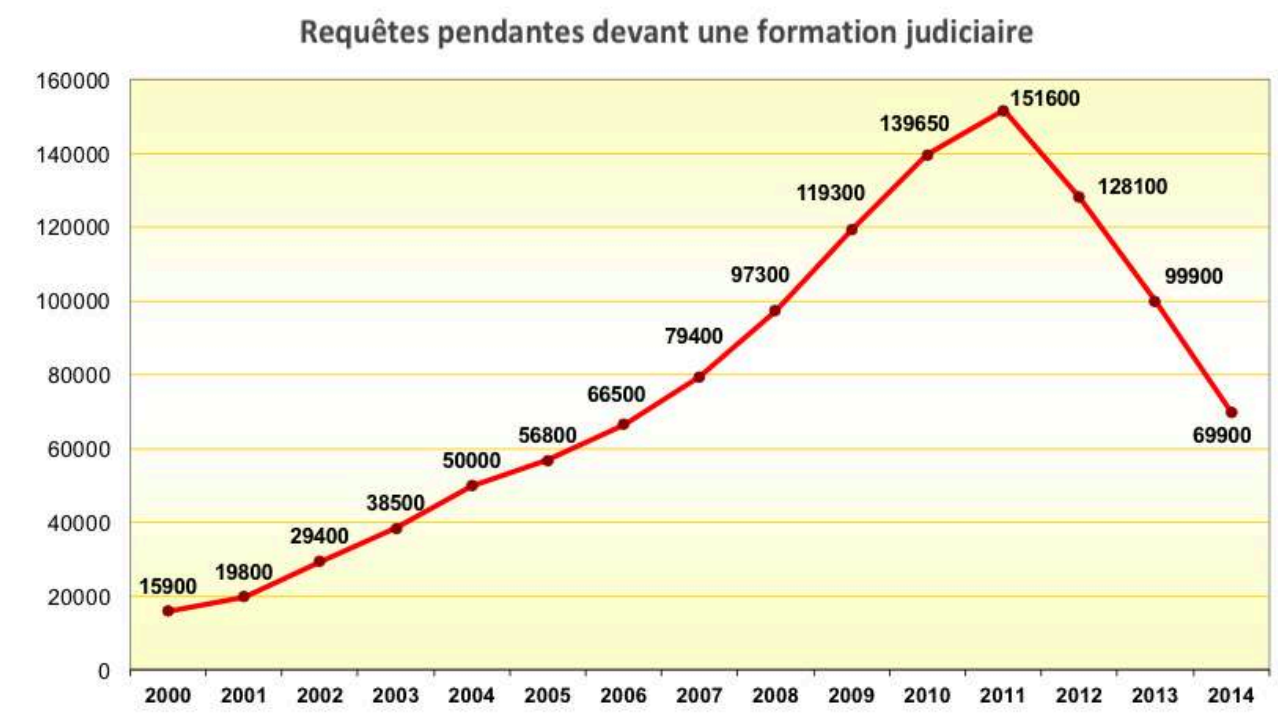

10 Ce résultat s'explique bien sûr par les importantes réformes menées depuis plusieurs années ${ }^{11}$, en particulier via le Protocole $n^{\circ} 14$ dont le «succès $»^{12}$ est indéniable. Ainsi, dès l'année 2012, les nouveaux mécanismes de filtrages des requêtes - tels que les formations de juge unique, le comité de trois juges ou la section de filtrage - et la rationalisation des méthodes de travail au sein de la Cour ont donné leur pleine puissance ${ }^{13}$. A tel point que les autorités de la Cour espèrent «qu'en 2014, il n'y aura plus d'arriéré pour les affaires à juge unique $»^{14}$.

11 Ainsi, en 2014, 86063 requêtes ont été jugées après le prononcé d'un arrêt (2 388) ou au terme d'une décision d'irrecevabilité ou de radiation (83 675). Mais par comparaison avec 2013 , ce chiffre est significativement en baisse (moins $8 \%$ ). Une telle évolution de la productivité strasbourgeoise ne trouve pas sa source dans le nombre de requêtes communiquées au Gouvernement, lequel reste stable (7 897 au lieu de 7931 ), mais se situe plus en amont du processus de traitement contentieux des requêtes.

12 En effet, la plus grande particularité du bilan de l'année 2014 réside dans le net accroissement du nombre de requêtes terminées administrativement, c'est-à-dire avant même qu'elles ne soient attribuées à une formation judiciaire. Alors qu'en 2013, 13650 requêtes avaient ainsi été écartées au stade pré-judiciaire, en 2014, ce chiffre s'est élevé à 25100 requêtes soit une augmentation considérable de $\mathbf{8 4} \%$. Mécaniquement et corrélativement, une réduction de $15 \%$ des nouvelles requêtes 
allouées à une formation judiciaire a été enregistrée en 2014 (56 250 au lieu de 65800 en 2013).

13 Cette évolution est essentiellement dû à " un changement important dans les procédures de

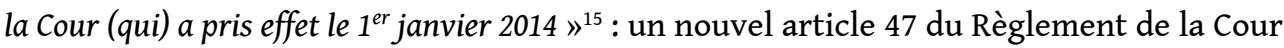
est entré en vigueur. Ce texte a fixé de nouvelles et plus strictes conditions de forme pour introduire une requête. Ceci, pour permettre à la Cour de disposer d'emblée "des informations suffisantes pour qu'elle puisse procéder à une première analyse de la requête ${ }^{16}$. Ainsi, et désormais, chaque requête doit être initiée par le biais d'un formulaire précis ${ }^{17}$ et être accompagnée des documents pertinents. A défaut, ladite requête ne pourra interrompre le délai de six mois pour saisir la Cour et risque, à terme, de s'exposer à une irrémédiable irrecevabilité ${ }^{18}$. En 2014, il en fut ainsi pour $23 \%$ des requêtes introduites ${ }^{19}$.

\section{2) Un reflux contentieux salvateur}

14 A n'en pas douter, un tel reflux quantitatif ne peut que soulager une Cour dont le risque d'asphyxie sous le poids des requêtes pendantes n'était pas fantasmé. Souligner que ces "résultat très positifs sont le fruit du travail énorme accompli au sein de la Cour par les juges et par les membres $d u$ greffe $»^{20}$ n'est d'ailleurs pas que simple rhétorique, puisqu'ils ont été atteints à moyens quasi-constants. Hormis quelques contributions volontaires d'Etat ${ }^{21}$, le budget de la Cour est ainsi demeuré au niveau réduit de 67,6 millions d'euros, ce qui demeure fort modeste au regard de l'immensité de la tâche ${ }^{22}$. En ces temps de disette budgétaire en Europe, le Président de la Cour ne l'a signifié que mezzo vocce ${ }^{23}$. Mais le Greffier Erik Fribergh a indiqué que seul un «budget extraordinaire » permettrait « d'absorber l'arriéré des affaires » ${ }^{24}$.

Quoiqu'il en soit, le bilan statistique pour l'année écoulée est encourageant. Non seulement il fait mentir certains des contempteurs du système européen des droits de l'homme qui avaient misé sur le risque d'asphyxie strasbourgeoise pour justifier une réduction drastique du pouvoir européen ${ }^{25}$. Mais au surplus, cette évolution permet à la juridiction strasbourgeoise d'affecter ses efforts et ressources à d'autres priorités.

16 L'une d'elles est de parvenir à un traitement plus rapide des requêtes ${ }^{26}$, dans le droit-fil du cap des «un an - deux ans " fixé à Brighton ${ }^{27}$. Une autre priorité est que la Cour puisse se concentrer sur les affaires les plus graves sans se noyer dans le flot d'« affaires répétitives ", lesquelles représentent « la moitié des requêtes pendantes (...), soit $35000{ }^{28}$ et sont plus que jamais le principal défi à relever de la juridiction européenne ${ }^{29}$. L'allègement de la charge contentieuse permet également à la Cour de consacrer plus d'énergie et de temps pour parfaire son œuvre jurisprudentielle ${ }^{30}$. Et ce, tout particulièrement via la formation solennelle de Grande Chambre ${ }^{31}$.

17 Il est donc manifeste que les enjeux quantitatifs sont étroitement corrélés aux enjeux qualitatifs. Et au demeurant, les succès statistiques seraient vides de sens s'ils ne contribuaient pas à accroître l'efficience du système européen des droits de l'homme.

\section{B - Le prisme qualitatif : Une ambivalente embellie contentieuse}

Que la Cour européenne des droits de l'homme soit résolument sur la voie de la résorption du stock de requêtes en souffrance ne peut que réjouir. Mais comme l'an passé $^{32}$, le bilan annuel n'est pas exempt de points noirs. Et ce, tant au regard des 
résultats pour le moins contrastés de certains Etats (1), qu'en raison des interrogations qui persistent concernant le libre accès des justiciables au prétoire européen (2).

\section{1) Des résultats étatiques contrastés}

La situation de certains Etats parties est encore loin d'être idyllique, comme en atteste le classement des plus gros pourvoyeurs de requêtes ${ }^{33}$. En 2014, l'Ukraine a le triste honneur de la première place avec 19,5\% des requêtes pendantes (soit 13650 requêtes), suivie de l'Italie à 14,4 \% (10 100 requêtes). Non sans ironie géopolitique, la Fédération de Russie et l'Ukraine ont échangé leur place respective. En effet, après avoir longtemps occupé la tête du classement, la Russie se situe désormais à la troisième place avec $14,3 \%$ du total (9 990 requêtes). La spectaculaire évolution russe était toutefois prévisible, car dès l'an passé, un mouvement de forte réduction des requêtes pendantes était déjà perceptible ${ }^{34}$. Gageons que l'Italie - dont les efforts actuels ont été salués par le Président Spielmann ${ }^{35}$ - empruntera un chemin identique.

20 L'exemplarité étatique n'est pas non plus de mise sur le front des violations constatées au cours de l'année 2014. Certes, à l'instar de la tendance observée l'an passé3 ${ }^{36}$, le nombre d'arrêts ${ }^{37}$ constatant au moins une violation a légèrement décru, passant de 797 à 756 . Certes également, le nombre de requêtes rayées du rôle à la suite d'un règlement amiable ou d'une déclaration unilatérale de l'Etat a significativement augmenté en $2014^{38}$, ce qui est en principe positif car implique une reconnaissance étatique de la violation et sa prompte réparation.

Mais au premier rang des condamnations figure toujours le non respect d'exigences procédurales (droit au procès équitable - 258 cas - et droit à un recours effectif 128 cas), ce qui induit l'existence de failles structurelles persistantes au sein des dispositifs nationaux. Plus inquiétant, la recrudescence des constats de violation au titre de l'article 3 (interdiction de la torture et des traitements inhumains et dégradants) observée en 2013 - avec 241 cas - s'est poursuivie en 2014, avec pas moins de 248 arrêts de violation rendus sur ce terrain. Cumulées avec les 69 condamnations au titre de l'article 2 (droit à la vie), les 5 au titre de l'article $4 \mathrm{du}$ Protocole $\mathrm{n}^{\circ} 7$ (droit à ne pas être jugé ou puni deux fois) ainsi qu'à celle au titre de l'article 7 (pas de peine sans loi), ce sont plus de $42 \%$ des constats de violations qui sont ainsi relatifs à des droits absolus au sens de l'article 15 .

Le triste palmarès des Etats les plus condamnés à Strasbourg demeure, quant à lui, inchangé. En 2014, la Russie et la Turquie conservent les premières et deuxièmes places (respectivement 122 et 93 arrêts constatant au moins une violation), toujours suivis de la Roumanie (74). Seule la Hongrie surgit (49) pour dépasser l'Ukraine et l'Italie (39 chacune).

Par contraste, le bilan européen de la France est moins calamiteux. Pour 1142 requêtes dûment introduites en 2014 (soit un ratio de 0,17 requêtes pour 10000 habitants), 1273 ont été déclarées irrecevables ou rayées du rôle. 17 des 22 arrêts rendus contre cet Etat se sont soldés par au moins un constat de violation. Fait notable, c'est au titre du droit au respect de la vie privée et familiale (Art. 8) que la France a été le plus souvent condamnée en 2014, à six reprises ${ }^{39}$. Viennent ensuite la liberté de réunion et d'association (Art. 11) et le droit à la liberté et à la sûreté (Art. 5) qui ont respectivement donné lieu à quatre et trois constats de violation. Mais s'agissant de l'interdiction de la torture et des traitements inhumains et dégradants 
(Art. 3), la France a connu une nette embellie en $2014^{40}$ avec seulement un arrêt portant constat de violation et une violation conditionnelle ${ }^{41}$.

Quoiqu'il en soit, nul ne saurait déduire de la nette réduction du volume global des affaires pendantes à Strasbourg une amélioration corrélative de la situation des droits de l'homme en Europe. Il est d'ailleurs symptomatique qu'en dépit de la persistance de nombreux défis pour les droits et libertés, l'année 2014 ait donné lieu à une inédite et significative diminution des «requêtes entrantes » dûment attribuées à une formation judiciaire ${ }^{42}$.

\section{2) L'enjeu persistant du libre accès au prétoire européen}

Inévitablement, se pose la question des conditions d'accès des requérants au prétoire de la Cour européenne. En effet, les statistiques de l'année 2014 pourraient donner crédit à une double hypothèse : d'une part, le goulot d'étranglement à l'entrée de la Cour constitué par la phase pré-judiciaire ${ }^{43}$ puis pré-communication ${ }^{44}$ s'est encore resserré; d'autre part, et corrélativement, ce durcissement emporterait un effet dissuasif sur les requérants potentiels qui renoncent ainsi à saisir la Cour ${ }^{45}$.

Pourtant, la nécessité que la juridiction strasbourgeoise ne "perde pas en légitimité et effectivité ce qu'elle a gagné en efficacité et productivité »" est toujours d'actualité. Bien sûr, à l'orée de l'année 2015, il apparaît assez nettement que la course effrénée au rendement statistique n'a pas conduit la Cour européenne à verrouiller l'accès à son prétoire en faisant totalement fi de sa raison d'être : l'humain et la protection de ses droits.

Il est toutefois indéniable que l'entrée en vigueur du nouvel article 47 du Règlement a bousculé quelques habitudes. Certes, les services strasbourgeois ont redoublé d'efforts pour expliciter ces nouvelles exigences formelles, en particulier sur le site internet de la Cour qui - cette année encore - conserve son statut de point de convergence numérique du système européen des droits de l'homme ${ }^{47}$. Certes également, il est légitime qu'un minimum de rigueur soit exigé des requérants et de leurs conseils à l'heure pour eux de solliciter la Cour.

Mais la vigilance doit rester de mise, notamment devant la montée en puissance d'une logique administrative de traitements des recours au détriment de la logique judiciaire. En effet, à la faveur du nouvel article 47 du Règlement, nombre de requêtes peuvent être écartées par les membres du Greffe sans qu'un juge ne se prononce. Par ailleurs, les mêmes membres du Greffe jouent un rôle déterminant lorsqu'ils aiguillent une requête vers le juge unique, et donc vers son rejet lapidaire par lettre type. Or, en pratique, et eu égard au volume des affaires ainsi traitées, le regard du juge est bien souvent réduit à une pure formalité.

Un mouvement similaire est identifiable dans la pratique strasbourgeoise des mesures provisoires, dont les fluctuations ont déjà suscité quelques critiques par le passé ${ }^{48}$. En 2014, l'augmentation - quelque peu en trompe l'œil ${ }^{49}$ - de mesures accordées par la Cour ne doit pas occulter le fait que presque $50 \%$ des demandes ont donné lieu à un rejet par le seul Greffe et sans soumission à un juge au motif qu'elles "ne relevaient pas du champ d'application de l'article 39 du règlement $\aleph^{50}$. Or, à l'insigne différence des requêtes, le traitement des demandes de mesure provisoire n'est guère encadré par des conditions textuelles précises, ce qui laisse une ample latitude au Greffe pour décider sans aucune regard juridictionnel - d'éluder cette demande. 

fil des nombreuses réflexions sur l'avenir de la Cour et réformes subséquentes, l'idée de confier à des membres du Greffe "l'exercice de certains pouvoirs judiciaires ${ }^{51}$ a toujours été rejetée, du moins formellement. Il est donc curieux qu'un tel dessaisissement au détriment des juges ait lieu de manière insidieuse, si ce n'est quasi-clandestinement.

Au-delà, c'est l'ensemble de cette phase cruciale d'examen initial des requêtes qui mérite plus d'attention. Car elle constitue la partie immergée et donc invisible de l'iceberg contentieux à Strasbourg. De fait, la grande majorité des requêtes initiées est écartée par une clôture administrative ${ }^{52}$ ou une décision de juge unique. Or, ces actes ne reçoivent guère de publicité et ne sont pas motivés, sauf de façon stéréotypée, ce qui fait obstacle à l'existence d'un regard extérieur susceptible de sonner l'alerte sur des dérives ou, plus simplement, une erreur d'appréciation ${ }^{53}$.

A l'évidence, ces derniers mois, les autorités strasbourgeoises semblent avoir pris la mesure de cette lacune de nature à miner gravement la confiance des justiciables envers la Cour ${ }^{54}$. Et donc, à terme, de saper la légitimité même de la juridiction européenne.

Ainsi, et d'une part, après avoir exprimé une réticence répétée envers l'idée d'une motivation des décisions d'irrecevabilité de juge unique ${ }^{55}$, le discours européen tend à évoluer significativement. En ce sens, il y a quelques jours, le Président Spielmann a esquissé la possibilité d'une "motivation, même brève, des décisions d'irrecevabilité de juge unique (...) une fois réglé le problème du "backlog" ». Ceci, afin de "répondre favorablement à (une) demande qui correspond à une attente légitime des requérants » et qui " aurait d'ailleurs une valeur pédagogique ", puisqu' "une motivation, même brève, aura un effet dissuasif sur les requêtes dénuées de chance de succès $»^{56}$.

D'autre part, à cet effort espéré de transparence individuelle pourrait s'ajouter une amélioration de la transparence collective avec la publication de statistiques plus fines concernant la phase initiale de traitement des requêtes et celles des travaux des organes de suivi interne chargés d'évaluer la mise en œuvre du nouvel article $47 \mathrm{du}$ Règlement. En ce sens, un « renforce(ment de) la communication sur cette disposition » a été annoncé ${ }^{57}$.

De telles évolutions seraient tout à fait louables, car elles réduisent le risque que la logique de réduction quantitative en vienne à primer exagérément sur la démarche qualitative. Mais il importe aussi que la cour agisse pour renforcer l'emprise judiciaire sur la phase de traitement administrative des requêtes. A cet égard, l'existence d'un droit de regard personnel du Président sur les 4 cas limites $»^{58}$ est une initiative utile. Mais elle mériterait d'être institutionnalisée - pour que ces bonnes intentions soient pérennisées au-delà du mandat de l'actuel Président - et même d'être étendue sous la forme d'un dispositif plus vaste d'alerte et de vigilance ${ }^{59}$.

36 En tout état de cause, les quelques zones d'ombre qui obscurcissent le bilan contentieux de l'année écoulée ne sauraient occulter combien celui-ci est positif. Or, cette indéniable victoire sur le front statistique est d'autant plus précieuse qu'elle permet à la Cour européenne de rassembler ses forces pour mener aujourd'hui d'autres batailles, non moins périlleuses.

La Revue des droits de l'homme, Actualités Droits-Libertés 


\section{$2 \%$ - Un présent assombri : Les oscillations du centre de gravité des droits de l'homme en Europe}

37 S'affirmer comme « centre de gravité de l'Europe des droits de l'homme » ${ }^{60}$. Indéniablement audacieux, cet objectif est pourtant à la portée de la Cour de Strasbourg. Mais le chemin pour y parvenir est semé d'embûches. Or, à cet égard, si nul ne peut être surpris des résistances étatiques opposées à de telles ambitions (B), il en est bien différemment de la retentissante opposition qui, aux dernières lueurs de l'année 2014, a surgi de la Cour de Luxembourg (A).

\section{A - Un regrettable coup d'arrêt porté à l'adhésion de l'Union européenne à la Convention}

«Je t'aime, moi non plus ». Ces quelques mots déjà employés par le passé pour décrire les relations entre les deux juridictions européennes ${ }^{61}$ n'ont pas perdu de leur actualité. Le retentissant avis rendu le 18 décembre 2014 par la Cour de justice de l'Union européenne sur le projet d'adhésion de l'Union à la Convention européenne des droits de l'homme ${ }^{62}$ en offre une éloquente illustration. Certes, à l'instar de toute relation passionnelle, l'idylle européenne entre Strasbourg et Luxembourg n'est jamais à l'abri de quelques passades orageuses et conflictuelles. Mais jusqu'à ce jour, chaque tension avait pu être surmontée sous les auspices de l'entente cordiale. Or, force est de constater que l'avis de décembre 2014 révèle cette fois une profonde crise conjugale (1), mue par une sourde lutte de pouvoir (2).

\section{1) Le retentissant veto de la Cour de justice de l'Union européenne}

39 Maintes fois décrite comme un véritable « serpent de mer » ${ }^{63}$ institutionnel, l'ambitieux projet d'adhésion de l'Union européenne à la Convention européenne des droits de l'homme a certes déjà connu son lot de retards, contestations et autres désillusions. Au demeurant, la lenteur du processus s'expliquait aisément, tant son importance le dispute à sa complexité64. Mais en avril 2013, un pas décisif semblait avoir été franchi avec l'adoption d'un projet d'accord d'adhésion ${ }^{65}$. Cependant, le chemin vers son adoption définitive par l'ensemble des Etats parties à la Convention passait par Luxembourg.

40 En effet, avant de pouvoir être approuvé par décision unanime du Conseil de l'Union européenne puis par les vingt-huit États membres "conformément à leurs règles constitutionnelles respectives "66, ce projet d'accord devait d'abord recueillir un avis positif de la part de la Cour de justice de l'Union européenne ${ }^{67}$. Or, tel ne fut pas le cas. Réunie en assemblée plénière, la Cour de Luxembourg a estimé que pas moins de sept points $^{68} \mathrm{du}$ projet d'accord étaient incompatibles avec l'article $6 \S 2$ du Traité sur l'Union européenne ${ }^{69}$ et à son protocole $\mathrm{n}^{\circ} 8$ relatif à l'adhésion de l'Union à la Convention.

41 Sans surprise, un tel avis a d'ores et déjà fait abondamment couler l'encre doctrinale et ce flot n'est sans doute pas près de se taririo71. Il serait donc vain en ces lieux de se 
livrer à une exégèse détaillée de l'avis de décembre $2014^{72}$. Pour autant, il n'est pas inutile de s'attarder sur les présupposés qui irriguent la position - "délibérément politique ${ }^{73}$ - retenue par des juges de Luxembourg, tant ils jettent une lumière crue sur l'avenir troublé du processus d'adhésion de l'Union à la Convention.

En effet, c'est essentiellement à la lueur de "la circonstance que l'Union est dotée d'un ordre juridique d'un genre nouveau, ayant une nature qui lui est spécifique, un cadre constitutionnel et des principes fondateurs qui lui sont propres, une structure institutionnelle particulièrement élaborée ainsi qu'un ensemble complet de règles juridiques qui en assurent le fonctionnement $»^{74}$ que la Cour de justice a évalué l'accord d'adhésion. Plus encore, précisément aux fins de "garantir la préservation des caractéristiques spécifiques et de l'autonomie de cet ordre juridique ", les juges du plateau de Kirchberg ont aussi insisté d'emblée sur l'existence d'un « système juridictionnel destiné à assurer la cohérence et l'unité dans l'interprétation du droit de l'Union $»^{75}$. Lequel est dominé par la Cour de justice elle-même.

Face à la "question centrale de la solubilité du système communautaire dans une juridiction internationale $»^{76}$, la Cour de justice a donc "magnifié la spécificité du droit de l'Union $»^{77}$ et ostensiblement affiché une forme d'irréductibilité du système de l'Union européenne. Et ce, jusqu'à forcer quelque peu le trait du particularisme unioniste. Ainsi, il est tout à fait significatif que la Cour de Luxembourg ait abondamment usé d'un vocable à coloration constitutionnalisante, telle l'expression "charte constitutionnelle» pour désigner les traités ${ }^{78}$ ou celle de "structure constitutionnelle de l'Union $»^{79}$. Or, non sans ironie, cette inclinaison sémantique n'est pas sans susciter quelques contradictions. En effet, quelques lignes auparavant, la Cour de justice avait tenu à affirmer que "l'Union, du point de vue du droit international, ne peut pas, en raison de sa nature même, être considérée comme un État ${ }^{80}$.

La contradiction n'est qu'apparente, puisque l'ensemble de ces éléments convergent pour affirmer l'irréductibilité de l'Union devant le système conventionnel. Mais ce faisant, la Cour de justice donne corps à une certaine forme de "souverainisme unioniste" qui ne jurerait pas avec les arguments dérivés du souverainisme national. Car en définitive, la juridiction de Luxembourg en vient à se draper dans des principes et arguments dont les ressorts sont identiques à ceux si souvent opposés à l'Union européenne par certains de ses contempteurs nationaux.

\section{2) Un avenir européen durablement hypothéqué}

L'unionisme, nouvel avatar post-moderne du nationalisme? La question est volontairement provocatrice.

Mais elle est moins fantaisiste qu'elle n'y paraît, tant l'avis du 18 décembre 2014 révèle une nette réticence de la Cour de justice à l'idée même d'un contrôle externe de l'Union par la Cour européenne des droits de l'homme ${ }^{81}$. Bien sûr, cette réticence est revêtue d'un habillage juridique qui, à défaut d'emporter l'approbation, permet à la Cour de Luxembourg de justifier sa position. Ainsi, il est indéniable que le Protocole $\mathrm{n}^{\circ} 8$ au TUE offrait un support à l'idée selon laquelle l'adhésion de l'Union à la Convention ne saurait froisser à l'excès «les caractéristiques spécifiques de l'Union et du droit de l'Union " Il n'est pas davantage contestable que certaines prises de positions passées de la Cour de justice pouvaient laisser augurer une telle issue ${ }^{83}$. favorable sous réserve de modifications mineures démontrent qu'une part substantielle des 
objections soulevées pouvait être surmontée par quelques aménagements et une lecture compréhensive des traités communautaires $»^{84}$. Il est tout aussi symptomatique que l'ensemble des commentateurs de l'avis, qu'ils soient ou non favorables à l'adhésion de l'Union à la Convention, aient estimé que le choix de la Cour de justice s'explique bien plus par son opposition au principe de l'adhésion, que par de quelconques imperfections techniques de l'accord ${ }^{85}$.

De fait, à force d'exacerber l'accessoire - i.e. la préservation des caractéristiques de l'Union par le projet d'adhésion -, la Cour de justice en a oublié le principal : l'adhésion de l'Union à la Convention, laquelle est gravée dans le marbre des traités fondateurs et procède d'un choix univoque des Etats membres de l'Union. En d'autres termes, «dans ses considérations liminaires » qui ont irrigué l'ensemble de son avis, la Cour de justice n'a "fait aucune place aux raisons qui ont milité en faveur de l'adhésion et au système de Strasbourg ${ }^{86}$ et n'a pas davantage été sensible au fait que "l'ensemble des Etats membres et des institutions européennes s'étaient déjà exprimés en faveur de la compatibilité de l'accord avec les traités sur l'Union européenne et sur le fonctionnement de l'Union européenne $»^{87}$

Dans ces conditions, s'il est vrai que "l'avis de la Cour de justice ne rend pas (l)e projet (d'adhésion) obsolète » et "ne lui fait pas perdre sa pertinence " ${ }^{88}$, il n'en reste pas moins que le veto de la Cour de justice sera bien difficile à surmonter tant les ressorts de sa décision se heurtent frontalement à l'idée même d'adhésion. Dès lors, sauf à ce que les Etats membres de l'Union décident de procéder à « un lit de justice européen » et brise la position de la Cour de justice en modifiant les traités fondateurs - ce qui est peu probable -, l'avis de décembre 2014 laisse augurer une lente et périlleuse renégociation de l'accord d'adhésion, déjà acquis de haute lutte ${ }^{89}$.

Quoiqu'il en soit, ce coup d'arrêt porté au processus d'adhésion - potentiellement synonyme de report "aux calendes grecques $»^{90}$ - ne pas sera pas sans conséquence. Indéniablement, « les premières victimes de l'avis $2 / 13$, ce sont les citoyens qui se voient ainsi privés du droit de soumettre les actes de l'Union européenne au même contrôle externe du respect des droits de l'homme que celui qui s'applique à tous les Etats membres $»^{91}$. Mais il est probable que l'Union européenne et sa Cour de justice en paient aussi le prix. Outre le coup porté à leur réputation ${ }^{92}$ - par contraste avec leur bilan remarquable en termes de protection des droits fondamentaux -, il n'est pas impossible que "l'Empire (strasbourgeois) contre-attaque ", pour reprendre la plaisante expression de Tobias Lock ${ }^{93}$.

51 Ainsi, en réaction à l'avis de la Cour de justice, le Président Dean Spielmann n'a pas seulement fait part de sa "grande déception». Presqu'en compensation de l'absence persistante d'un contrôle externe de l'Union, il a également souligné que "plus que jamais (...), il s'agira pour notre Cour de protéger au mieux les citoyens des effets négatifs de cette situation, dans le cadre des affaires qui lui seront soumises ${ }^{94}$. Or, s'il importe de ne pas extrapoler cette assertion glissée au détour du bilan annuel, elle pourrait préfigurer un durcissement de la jurisprudence strasbourgeoise ${ }^{95}$, en particulier via un affaiblissement de la "présomption de conventionalité » concédée jusqu'à ce jour à l'Union ${ }^{96}$. En d'autres termes, face à l'adhésion sans cesse repoussée, la Cour européenne des droits de l'homme pourrait chercher - à rebours des espoirs de la Cour de justice - à conforter un peu plus encore la Convention dans son statut de texte « de droit commun des droits fondamentaux en Europe $"{ }^{97}$.

52 Preuve supplémentaire de résilience face à l'adversité, cette fermeté strasbourgeoise teintée d'habilité a d'ailleurs déjà fait ses preuves face aux critiques étatiques. 


\section{B - Une perpétuelle lutte européenne face aux résistances étatiques}

53 Nul n'en sera surpris : les vives critiques qui ciblent le système européen des droits de l'homme depuis désormais quelques années ${ }^{98}$ ne se sont pas estompées en 2014 (1). En réaction, la vigilance et le sang-froid devront être les maitres-mots de la Cour en 2015 (2).

\section{1) Des critiques étatiques renouvelées}

Bien évidemment, les attaques étatiques ne sauraient être ignorées, tant elles sont susceptibles d'affaiblir singulièrement l'autorité du système européen des droits de l'homme.

Il en est particulièrement ainsi lorsqu'elles proviennent d'Etats fondateurs tels que le Royaume-Uni. Outre-Manche, les récurrentes critiques du Gouvernement conservateur de David Cameron ${ }^{99}$ ont redoublé d'intensité à mesure que se rapprochent les élections de mai 2015. Ainsi, notamment par la voix de l'actuel - et fort peu europhile - ministre de la Justice, Chris Grayling, un véritable " plan " pour " changer le droit britannique des droits de l'homme $»^{100}$ a été rendu public en octobre 2014. Censé être mis en œuvre par le futur Gouvernement conservateur en cas de victoire électorale, ce projet prend la forme d'une alternative pour le moins offensive: soit une réduction drastique de l'autorité de la Cour européenne au Royaume-Uni est entérinée, en particulier avec la reconnaissance d'un «droit de veto » du Parlement britannique qui disposerait alors du "dernier mot »; soit le Royaume-Uni se retire de la Convention européenne ${ }^{101}$.

A l'évidence, cette alternative est en trompe l'œil. Car l'idée même de «droit de veto » national ou de «neutralisation démocratique » des décisions de la Cour - déjà évoquée par le passé ${ }^{102}$ - serait en contradiction frontale avec le système européen des droits de $l^{\prime}$ homme ${ }^{103}$. Selon le projet conservateur, le Royaume-Uni ne résisterait donc à la tentation de quitter ce système européen que s'il parvient à en saper les fondements et à le réduire au silence ${ }^{104}$. Bien sûr, ce projet soulève nombre de contestations et objections ${ }^{105}$, jusqu'au sein même du parti conservateur ${ }^{106}$ et de son allié Lib-Dem ${ }^{107}$. Au surplus, nul n'ignore que le "Strasbourg-bashing $»^{108}$ outre-Manche se nourrit dans une large mesure des craintes électorales du parti conservateur d'être débordé sur sa droite par le parti UKIP. Enfin, un tel « dilemme britannique face à la Cour européenne des droits de l'homme » n'est aucunement inédit dans l'histoire du royaume d'outre-Manche ${ }^{109}$.

Néanmoins, la perspective d'une dénonciation de la Convention européenne par le Royaume-Uni est devenue tangible ces derniers mois, alors qu'elle était encore chimérique il y a quelques années. Surtout, chaque geste britannique esquissé vers la sortie du système européen accroît le risque de contagion ${ }^{110}$. Ainsi, ces critiques proférées à l'Ouest de Strasbourg confortent celles, non moins incisives, émises à l'Est : en Russie, plusieurs voix - dont celle du Président Poutine - ont évoqué un retrait de la Convention européenne des droits de l'homme ${ }^{111}$. Plus récemment encore, le conflit entre la Russie et l'Ukraine exacerbe les tensions et ravive les menaces d'un départ russe du Conseil de l'Europe ${ }^{112}$. Moins pressants, mais tout aussi récurrents, sont les appels au retrait lancés en Suisse ${ }^{113}$. La France n'a pas non été épargnée par de telles saillies ${ }^{114}$, au lendemain de quelques condamnations sur des sujets sensibles ${ }^{115}$. 


\section{2) Les impératifs européens de vigilance et de sang-froid}

58 Face à ces critiques, réaffirmer que toute dénonciation de la Convention par un Etat partie «serait toujours un très grand échec pour la protection des droits fondamentaux en Europe » et "un véritable désastre politique »116 est évidemment plus qu'opportun. Mais il importe aussi de ne pas extrapoler la menace, ni de lui donner plus d'importance qu'elle n'en a réellement.

59 D'une part, les critiques - bien souvent tonitruantes - visant le système européen des droits de l'homme ne doivent pas occulter les indéniables soutiens dont il jouit. En ce sens, même dans des Etats où les contempteurs sont légions, il est stratégiquement plus utile d'insister sur les succès et les progrès, plutôt que de prêter attention aux critiques populistes imperméables aux arguments rationnels ${ }^{117}$. En agissant ainsi, la Cour apparaît comme une alliée ouverte à l'échange et ses ennemis sont réduits à ce qu'ils sont : essentiellement une minorité, fût-elle parfois plus bruyante que la majorité.

60 De même, il est aussi opportun d'insister sur les incontestables succès engrangés par la Cour. Et ce, même sur des sujets éminemment brûlants. Ainsi, concernant la gestation pour autrui, les réactions politiques exacerbées en France contrastent singulièrement avec l'attitude des plus hautes juridictions nationales. Car au lendemain des arrêts Mennesson et Labassée c. France, ces juridictions en ont promptement tiré les conséquences $^{118}$. De même, à propos des arrêts Matelly et ADEFDROMIL relatifs aux syndicats dans l'armée ${ }^{119}$, plutôt que de choisir la voie de la contestation frontale - et donc stérile -, les autorités ont souhaité accepter la solution européenne afin d'en tirer le meilleur profit ${ }^{120}$. Même le Royaume-Uni donne à voir de telles réactions constructives. Ainsi, au moment même où certains hauts magistrats britanniques ont suggéré de contrebalancer l'influence européenne par les normes constitutionnelles nationales ${ }^{121}$, de remarquables et fructueux échanges - par arrêts interposés - ont eu lieu entre la Cour européenne et la Cour suprême du Royaume-Uni ${ }^{122}$.

61 Ces exemples illustrent à l'envi combien le dialogue entre la Cour et les autorités nationales - en particulier les juridictions suprêmes - peut être extrêmement fructueux ${ }^{123}$. Et ce, malgré l'hystérisation de certains discours politiques.

D'autre part, et une fois de plus, "les vives réactions et critiques, aussi outrancières soientelles, sont aussi les symptômes d'une action utile »"24. En effet, l'intensification des attaques atteste surtout d'un accroissement de l'autorité de la Cour européenne ${ }^{125}$. Plus encore, c'est aussi parce qu'ils sont «frustrés de voir leurs juridictions nationales suivre la Cour de Strasbourg dans d'importantes affaires» que nombre d'«acteurs politiques cherchent à accroitre la distance entre les ordres juridiques nationaux et le droit conventionnel, ouvrant ainsi de l'espace pour une contestation politique $»^{126}$.

63 Partant, si mener "une réflexion globale de tous les acteurs au niveau européen, sans démagogie ni simplifications abusives $»^{127}$ sur les équilibres du système européen des droits de l'homme n'est jamais inutile, les juges européens doivent aussi prendre conscience que nombre de critiques étatiques sont irréductibles. Et que nombre de contempteurs sont bien plus prompts à prendre toute concession européenne pour une faiblesse, et toute condamnation strasbourgeoise pour une provocation ${ }^{128}$. 
Face à l'adversité, la fermeté et la cohérence sont donc également des vertus à cultiver. Car la seule prudence - souvent teintée de pusillanimité - est parfois contreproductive $^{129}$. Ainsi perçue, la résilience juridictionnelle est donc une force susceptible de renforcer l'autorité et la légitimité de la Cour. Or, ce trait de caractère ne sera pas superflu à l'heure d'aborder un futur européen riche en défis.

\section{$3 \%$ - Un futur riche en défis : Vers un nouveau chapitre de l'histoire de la Cour européenne}

65 A l'exacte image de $2012^{130}$, l'année qui commence peut aisément être qualifiée d'« année charnière » pour la Cour européenne des droits de l'homme. En effet, au cours de l'année 2015, la composition et la physionomie de la juridiction strasbourgeoise seront significativement renouvelées (A). Au surplus, la Cour devra affronter une nouvelle Conférence dédiée à son avenir. Cependant, à l'insigne différence de la Conférence de Brighton, ce sont cette fois les Etats qui se trouvent essentiellement sur la sellette (B).

\section{A - Le défi du renouvellement de la juridiction européenne}

"Qu'on le veuille ou non, que cela plaise ou non, la Cour de Strasbourg ne pourra pleinement devenir le juge suprême des droits de l'homme en Europe (...) qu'à condition de profiter d'un recrutement privilégiant (...) l'excellence ${ }^{131}$. Ecrits il y a près de quinze ans par le regretté Professeur Flauss, ces mots n'ont aucunement perdu de leur actualité. Bien au contraire, plusieurs travaux ont amplement confirmé combien sont étroits les liens entre la composition de la Cour et sa légitimité132. Au surplus, nul ne peut ignorer l'impact de l'équation personnelle sur les équilibres jurisprudentiels, l'influence d'un seul juge pouvant être déterminante, surtout lorsqu'il exerce une position éminente au sein de la juridiction.

67 Dans ces conditions, l'année 2015 sera des plus cruciales tant le renouvellement humain qui s'annonce au sein du Palais des Droits de l'Homme sera de grande ampleur. En effet, le départ de plus d'un quart de l'effectif total de la juridiction strasbourgeoise sera synonyme d'élections de nouveaux juges européens (1), mais aussi de notables remaniements de l'organigramme interne (2).

1) Vers une composition renouvelée : Le chassé-croisé des juges européens

68 L'année 2015 va donner lieu à un impressionnant chassé-croisé juridictionnel puisque pas moins de quinze sièges ont vocation à être renouvelés. Et ce, au terme d'un processus désormais bien plus rigoureux, les autorités européennes ayant souhaité mettre fin aux excès et dérapages du passé ${ }^{133}$. Ainsi, à la phase nationale de sélection des trois candidats ${ }^{134}$ succède une phase européenne qui se décompose elle-même en plusieurs étapes : d'abord, un examen attentif de cette liste de trois candidats par un Comité consultatif d'experts indépendants puis par une sous-commission de 
l'Assemblée parlementaire; ce n'est qu'ensuite que l'Assemblée parlementaire du Conseil de l'Europe sera appelée à voter pour élire le juge parmi ces trois candidats ${ }^{135}$.

Or, il n'est plus rare que l'Etat partie essuie un rejet de sa liste par la sous-commission, si celle-ci estime que tout ou partie des candidats ne présente pas le niveau exigé pour siéger à Strasbourg. En 2011, la France en avait ainsi fait l'amère expérience ${ }^{136}$. Plus récemment, c'est par deux fois que la République Slovaque a vu sa liste rejetée ${ }^{137}$. Une telle sévérité est louable, tant «il est crucial que l'excellence soit le moteur de tout processus de désignation et d'élection de juges " $"$.138.

70 C'est donc sous ces auspices qu'aux premières lueurs de l'année 2015, deux juges ont d'ores et déjà désignés. Les $\mathbf{2 7}$ et $\mathbf{2 8}$ janvier, l'Assemblée parlementaire du Conseil de l'Europe a ainsi élu Yonko Grozev juge à la Cour au titre de la Bulgarie et Branko Lubarda juge à la Cour au titre de la Serbie, en remplacement - respectivement - des juges Zdravka Kalaydjieva ${ }^{139}$ et Dragoljub Popović ${ }^{140}$. En avril prochain, six autres juges seront désignés pour remplacer les juges Mark Villiger - Liechtenstein -, Isabelle Berro-Lefèvre - Monaco -, Ján Šikuta - République Slovaque - et George Nicolaou Chypre. L'Assemblée devra aussi pourvoir aux sièges attribués aux titres de l'Arménie et de l'Irlande, actuellement vacants. Enfin, en juin 2015, ce sont encore six nouveaux juges qui prendront la route de Strasbourg à la faveur du vote de l'Assemblée parlementaire. Et ce, afin de remplacer les juges Dean Spielmann - Luxembourg -, Josep Casadevall - Andorre -, Boštjan Zupančič - Slovénie -, Elisabeth Steiner - Autriche - et Khanlar Hajiyev - Azerbaidjan -, ainsi que pour désigner le juge élu au titre de la Lettonie dont le siège est vacant.

71 Impressionnante sur le plan quantitatif, cette vague de renouvellement méritera également d'être soigneusement examinée sous un prisme qualitatif. Certes, en principe et plus que jamais, le processus de sélection garantit un haut niveau de recrutement, ainsi qu'une représentation équilibrée entre juges masculins et féminins ${ }^{141}$. Mais d'autres facteurs sont susceptibles de faire significativement varier les équilibres au sein du Palais des Droits de l'Homme, telles la formation des juges, leurs expériences professionnelles passées et même leurs orientations idéologiques ${ }^{142}$.

72 D'aucuns soulignent d'ailleurs, pour le regretter, que la «configuration sociologique » de la Cour a bien évolué depuis ces premiers pas. Initialement composée de personnalités issues de "l'élite académico-juridique très bien connectée » avec les réseaux politiques, la juridiction européenne aurait fait l'objet d'un processus de « judiciarisation » accrue. Ceci, en accueillant des juges plus spécialisé dans le champ juridique, mais doté d'un «capital social et politique » moindre que par le passé ${ }^{143}$. Il en résulterait notamment une insuffisante prise en compte par la Cour « de la nature intrinsèquement politique des droits de l'homme " au profit de " solutions judiciairo-bureaucratique » ${ }^{144}$. Sévère, cette conclusion est aussi quelque peu discutable. Car ces dernières années, les juges strasbourgeois - et notamment leurs Présidents successifs - n'ont jamais omis d'inscrire leur action dans une perspective diplomatique ${ }^{145}$. Quoiqu'il en soit, cette lecture sociologique éclaire singulièrement l'importance du choix des femmes et des hommes ainsi appelés à siéger à Strasbourg.

73 Or, s'il en est ainsi s'agissant du recrutement externe de nouveaux juges, il n'en est pas différemment concernant la réorganisation interne du Palais des Droits de l'Homme. 


\section{2) Vers une physionomie renouvelée : La réorganisation de la direction européenne}

74 La vague de renouvellement juridictionnel va mécaniquement emporter un vaste remaniement interne. En effet, comme en $2012^{146}$, ce sont les mandats de juges titulaires d'importantes fonctions au sein de la Cour qui vont arriver à échéance en 2015.

75 Il en est ainsi, en tout premier lieu, de celui qui préside aux destinées de la juridiction strasbourgeoise depuis le $1^{\text {er }}$ novembre 2012 : Dean Spielmann. Le 31 octobre prochain, peu après avoir soufflé les bougies de son cinquante-troisième anniversaire, celui qui est devenu le plus jeune juge de l'histoire de la Cour européenne des droits de l'homme à occuper ces hautes fonctions présidentielles ${ }^{147}$ achèvera son mandat de juge élu au titre du Luxembourg. Par voie de conséquence, le "chef d'orchestre $»^{148}$ de la Cour qui, durant trois ans, a veillé à ce que la symphonie européenne se joue sans fausses notes et ce, non sans succès même si "l'heure du bilan n'est pas encore venue »" ${ }^{149}$ - remettra sa baguette dans les mains d'un ou une autre juge.

$76 \mathrm{Au}$ même moment, d'autres fonctions de tout premier plan à Strasbourg seront également à pourvoir avec la fin du mandat du juge andorran Josep Casadevall - vicePrésident de la Cour et président de la Troisième Section -, du juge du Lichtenstein Mark Villiger - présidente de la Cinquième Section - ainsi que de la juge monégasque Isabelle Berro-Lefèvre - présidente de la Première Section. A l'image de $2011^{150}$ et $2012^{151}$, l'Assemblée plénière de la Cour ${ }^{152}$ pourrait donc se réunir durant l'été afin de désigner les nouveaux titulaires de ces fonctions, en particulier celle de Président.

77 Mais en 2015, la mission élective de l'Assemblée ne se limitera pas aux seules fonctions strictement juridictionnelles. Avec le départ du Greffier, Erik Fribergh ${ }^{153}$, et du Greffier adjoint, Michael O'Boyle ${ }^{154}$ - chacun atteint par la limite d'âge de 65 ans pour les agents du Conseil de l'Europe - deux autres postes cruciaux dans le fonctionnement de la Cour européenne des droits de l'homme seront à pourvoir ${ }^{155}$.

78 A de nombreux égards, une page va donc se tourner à Strasbourg en 2015 et un nouveau chapitre de l'histoire européenne s'ouvrira alors. Mais l'écriture de l'actuel chapitre est encore loin d'être achevé. Surtout, il n'est pas douteux que la continuité institutionnelle primera sur les changements personnels.

\section{B - Le défi de l'autorité et de l'effectivité des décisions européennes}

«Comment rester puissante dans un environnement hostile? $»^{156}$. En peu de mots, telle est l'interrogation qui agite nombre de juridictions internationales ${ }^{157}$. Mais cette question prend une résonnance toute particulière à Strasbourg, tant la Cour affronte des critiques récurrentes qui confinent parfois à la fronde étatique. Pourtant, à l'heure où se profile la Conférence de Bruxelles, ce sont plutôt les Etats parties qui se trouvent face à leurs responsabilités (1). Et c'est envers eux que les exigences de prévention des violations et d'exécution des arrêts vont s'imposer, sous le regard attentif de la Cour européenne (2). 


\section{1) La Conférence de Bruxelles : Des Etats parties face à leurs responsabilités}

80 Certes, c'est surtout d'outre-Manche et de l'Oural que souffle le vent de contestation le plus régulier et violent ${ }^{158}$. Les quelques intempéries britanniques et russes ne doivent donc pas occulter le fait que, bien souvent, les relations européano-étatiques se développent sous des cieux plus cléments. Au surplus, même les Etats parties les plus véhéments respectent bien plus qu'il n'y paraît les règles du système européen des droits de l'homme ${ }^{159}$.

81 Les réticences étatiques - souvent épidermiques, parfois existentielles -n'en sont pas moins un défi crucial pour l'autorité de la Cour et l'effectivité de ses décisions. L'un des symptômes les plus aigus de ce phénomène est le refus frontal d'un Etat de se plier à la solution retenue par la juridiction européenne ${ }^{160}$. Mais bien plus fréquemment, c'est à bas bruit que se manifeste l'obstruction nationale. Ainsi, il n'est pas rare qu'à l'heure de donner à la solution européenne une pleine effectivité dans l'ordre juridique interne, les autorités étatiques se lancent dans une véritable course de lenteur.

82 Les conséquences d'une telle attitude sont notoires. D'une part, l'autorité de la Cour européenne elle-même en souffre, au risque d'affaiblir sa légitimité et donc son efficacité. D'autre part et surtout, face à l'impossibilité d'obtenir au plan interne la jouissance de droits et libertés, les justiciables se tournent logiquement vers Strasbourg afin d'obtenir satisfaction. Ainsi, lorsque la faille nationale est aussi béante que persistante, de nombreuses requêtes s'y engouffrent et viennent inutilement encombrer le rôle de la Cour européenne. D'où ce fléau qui s'abat sur Palais des droits de l'homme : le flot d'affaires répétitives.

83 La difficulté est connue et les multiples conférences internationales qui se sont penchées sur l'avenir du système européen des droits de l'homme n'ont jamais manqué de le souligner ${ }^{161}$. En outre, ces dernières années, la Cour n'a pas ménagé ses efforts pour tenter de réduire cette difficulté. Nombre de réflexions ont même été - et sont encore - menées à ce sujet ${ }^{162}$. Mais à ce jour encore, pas moins de 35000 requêtes répétitives sont en souffrance à Strasbourg, ce qui représente la moitié des requêtes pendantes ${ }^{163}$. Certes, la disparition programmée en 2015 de l'arriéré des affaires de juge unique ${ }^{164}$ va permettre à la Cour de concentrer ses efforts sur cet autre front des requêtes répétitives ${ }^{165}$. C'est ainsi que le Greffier de la Cour a pu «prédire qu'en 2015, il y aurait un très grand nombre d'affaires répétitives traitées » en particulier via la Section de filtrage ${ }^{166}$.

84 Mais pour reprendre les mots du Président Spielmann, "la Cour pourra adopter les méthodes de traitement les plus efficaces, la solution, s'agissant des affaires répétitives, se trouve généralement au niveau interne ». Ainsi, «c'est (à) chaque pays (de) faire en sorte que les problèmes endémiques soient résolus en interne plutôt que d'être portés devant la Cour ${ }^{167}$. De fait, aujourd'hui, les principales marges de progression se nichent bien plus au sein des ordres juridiques étatiques qu'au cœur du Palais des Droits de l'Homme.

Dès lors, après une succession de conférences internationales où tous les regards - plus ou moins bienveillants ${ }^{168}$ - se sont focalisés sur la Cour européenne et ses difficultés, l'heure est désormais à un examen attentif des actions et inactions des Etats parties ${ }^{169}$. En atteste ainsi l'organisation les 26 et 27 mars prochain d'une conférence de haut niveau à Bruxelles intitulé « la mise en cuvre de la Convention européenne des droits de l'homme, notre responsabilité partagée ". 

avantages : outre qu'ils évitent une sanction - toujours infamante - à Strasbourg, ils protègent au mieux les droits des personnes placées sous leur juridiction. De plus, ils évitent à la Cour d'être noyée par un flot de requêtes qui peut être aisément tarie à la source. Pour ce faire, les autorités administratives, législatives et juridictionnelles nationales doivent «s'assurer que leurs lois, (...) pratiques administratives (et jurisprudences) sont en conformité avec les principes qui sont développés dans la jurisprudence issue de la Convention $»^{178}$.

91 En effet, ainsi que l'a souligné Jennifer Marchand dans un récent et remarquable article, « la portée prospective de la jurisprudence de la Cour lui confere une autorité de nature à orienter l'ensemble des États vers un standard élevé en matière de respect des droits de l'homme, en leur imposant d'anticiper les réformes structurelles suggérées par les arrêts de la Cour $»^{179}$. Tel est d'ailleurs le sens de la jurisprudence européenne qui impose aux juridictions nationales de relayer eux-mêmes et sans délai les exigences conventionnelles, fût-ce au prix d'une interprétation neutralisante du droit national ${ }^{180}$. Dans le même sens, la pratique des "arrêts pilotes" et celle de "prioritisation " contribuent à identifier les problèmes systémiques afin de les résorber au plus vite. Il serait d'ailleurs opportun que la Cour persiste dans cette dynamique inventive en 
admettant l'idée - récemment émise par quarante-deux ONG - d'« un recours d'intérêt collectif, permettant aux associations de porter devant la Cour un litige mettant en jeu la fin sociale en vue de laquelle elles ont été constituées $»^{181}$.

Quoiqu'il en soit, l'année 2015 a amplement confirmé combien l'autorité de la jurisprudence européenne est de nature à être confortée par l'action nationale. Pour ne prendre qu'un seul exemple français, la Cour de cassation a admis pour la première fois qu'une condamnation de la France par la Cour européenne constituait un changement de circonstances de droit justifiant la transmission au Conseil constitutionnel d'une question prioritaire de constitutionnalité ${ }^{182}$. Plus récemment encore, la Cour de cassation est parvenue à la même conclusion en visant un arrêt rendu par la Cour de Strasbourg contre un Etat autre que la France ${ }^{183}$, donnant ainsi toute son ampleur à l'idée d'effet « erga omnes » des arrêts européens ${ }^{184}$.

93 Exécution des arrêts, ensuite, car comme cela fut rappelé lors de la conférence d'Oslo ${ }^{185}$, "l'impérieuse nécessité d'une pleine exécution des arrêts » ${ }^{186}$ conditionne la crédibilité du système européen des droits de l'homme. Annoncée comme "au centre des préoccupations des Etats membres $»^{187}$ lors de la Conférence de Bruxelles, cet enjeu demeure aussi au cœur de l'actualité en particulier via l'interminable saga du droit de vote des détenus au Royaume-Uni. En effet, ce 10 février, la Cour européenne a prononcé une énième condamnation ${ }^{188}$. Mais si les juges européens ont renouvelé leur appel à une évolution de la législation britannique - appel resté sans suite à ce jour ${ }^{189}$-, ils se sont abstenus de condamner le Royaume-Uni au versement de "satisfactions équitables ». Or, en refusant ainsi d'user du redoutable levier financier, la Cour prend le risque de donner le sentiment qu'elle "se lave les mains » de l'atteinte ainsi subie par les détenus ${ }^{190}$. Et donc qu'elle cède face à la résistance aussi hostile qu'obstinée du Gouvernement Cameron.

Quoiqu'il en soit, cet exemple paroxystique de refus d'exécution des arrêts confirme une fois de plus les insuffisances criantes du dispositif de surveillance de l'exécution des arrêts européens ${ }^{191}$ et la nécessité corrélative d' ' un net renforcement de l'efficacité d(e ce) système de surveillance $»^{192}$. A cet égard, tout comme en $2014^{193}$, les interrogations sur la capacité du Comité des ministres du Conseil de l'Europe - à qui cette mission de surveillance incombe prioritairement ${ }^{194}$ - n'ont pas cessé.

95 Une telle situation ne peut donc qu'attiser le désir de la Cour européenne de fouler pleinement ce terrain de l'exécution des arrêts, si crucial pour sa propre autorité. Certes, formellement, la juridiction strasbourgeoise se refuse toujours à renverser le dogme selon lequel "l'exécution des arrêts relève essentiellement des autorités nationales et $d u$ Comité des Ministres ${ }^{195}$. Mais elle brûle d'inciter ces derniers à plus de dynamisme ${ }^{196}$.

Pour ce faire, la Cour semble fermement décidée à poursuivre sur une voie déjà amplement explorée ${ }^{197}$ : agir pour rendre ses arrêts " auto-suffisants » - à défaut d'être formellement "self executing" au sein de l'ordre national - de façon à guider étroitement autant leur exécution par l'Etat concerné que la surveillance de cette exécution par le Comité des ministres. Dépassant quelque peu la seule technique des arrêts pilotes ${ }^{198}$, la juridiction européenne n'hésite plus à revendiquer son ambition plus vaste de «donn(er) des indications en vertu de l'article 46 de la Convention (afin) d'aider ou d'encourager les autorités nationales à prendre les mesures requises pour exécuter un de ses arrêts $»^{199}$.

Plus encore, elle suggère désormais d'intégrer cette question au cœur même du débat contentieux ${ }^{200}$. La manœuvre est fort habile, car ainsi, les mesures indiquées au terme 
d'un débat contradictoire disposeront d'une autorité plus forte encore. De plus, la Cour pourra étendre encore son emprise sur la phase d'exécution. Et ce, à rebours de la lettre de la Convention, mais en pleine conformité avec l'esprit du système européen des droits de l'homme dont le maître mot doit être l'effectivité ${ }^{201}$.

Il en serait parfaitement de même si la Cour persistait à progresser encore sur un autre enjeu crucial : "la possibilité pour un requérant de demander la réouverture de la procédure interne une fois que la Cour a conclu que la procédure initiale était contraire à la Convention $»^{202}$. Certes, à ce jour, les juges européens n'ont aucunement érigé cette réouverture en condition sine qua non de la bonne exécution d'un arrêt ${ }^{203}$, même en matière pénale ${ }^{204}$. Il n'en demeure pas moins que la Cour ne cesse d'encourager vivement les Etats à agir en ce sens ${ }^{205}$. En atteste ainsi le très récent arrêt de Grande Chambre Bochan c. Ukraine $\left(\mathrm{n}^{\circ} 2\right)$ où la Cour en est même venue - certes fort logiquement - à contrôler les conditions dans lesquelles une telle procédure de réouverture s'est déroulée au plan interne ${ }^{206}$.

99 A l'évidence, donc, le volontarisme est à l'ordre du jour à Strasbourg. Et ce, notamment afin de nourrir un processus de «judiciarisation et (de) dépolitisation parallèle du processus d'exécution $»^{207}$ des arrêts. Or, il y a lieu de se réjouir d'un tel mouvement, tant il est riche de promesses pour l'autorité de la Cour européenne.

\section{En guise de conclusion : La Cour européenne, dernière ligne de défense des droits de l'Homme}

100 «La dernière ligne de défense des droits de l'Homme, le dernier rempart »"208. C'est par ces mots qu'en 2013, Françoise Tulkens décrivait la Cour européenne des droits de l'homme dont elle fut la vice-Présidente. Or, à l'orée de l'année 2015, ce constat ne s'est nullement démenti. Bien au contraire, il mériterait d'être gravé au frontispice de l'édifice européen de protection des droits de l'homme.

Mais en 2015, cette lourde mission de la juridiction strasbourgeoise n'est guère aisée.

A de nombreux égards, en effet, le continent européen reste durement éprouvé par une profonde crise économique ${ }^{209}$ qui se décline en une multitude d'inquiétants symptômes : "crise de l'identité européenne, crise psychologique et éthique, crise politique, crise de confiance, crise culturelle (...), crise de solidarité, crise sociale, crise de la démocratie et de l'Etat de droit, crise environnementale, crise sécuritaire $»^{210}$.

Les tragiques attentats de Paris en janvier dernier et ceux commis à Copenhague il y a quelques heures renforceront sans nul doute les stigmates de cette crise protéiforme. Car la folie meurtrière de quelques-uns risque de nourrir des sentiments aussi funestes que la haine et la peur mêlée de l'autre. Toute honte bue, certains n'ont d'ailleurs pas hésité à tirer profit de l'instant pour souffler sur les braises de l'intolérance. Et d'autres - non moins opportunistes - en ont profité pour en relancer leurs attaques récurrentes et obsessionnelles contre le système européen des droits de l'homme ${ }^{211}$. 
104 Mais c'est précisément dans contexte troublé, où de nombreuses digues nationales menacent de céder, que la Cour européenne des droits de l'homme doit conserver, contre vents et marées, son rôle crucial de phare des droits de l'homme en Europe. En particulier, il est essentiel - si ce n'est vital - que la juridiction strasbourgeoise agisse résolument pour «maintenir la lutte contre le terrorisme sous l'emprise des droits fondamentaux $»^{212}$ et qu'elle demeure le « garant d'un ordre public pacifique en Europe $»^{213}$.

105 Pour ce faire, malgré l'adversité et la sensibilité des enjeux portés au cœur, la Cour dispose de nombreux atouts. Pour mener à bien sa mission et accroître sa légitimité juridique, politique mais aussi sociétale ${ }^{214}$, il lui revient d'user autant de pédagogie et dialogue que de fermeté et de cohérence ${ }^{215}$, tout particulièrement dans ses relations avec les acteurs étatiques.

106 Pédagogie et dialogue, d'abord, car il est indéniable que le système européen des droits de l'homme "repose sur un partage de responsabilité global et dialogique entre les autorités nationales et la Cour». Selon les mots de Jean-Marc Sauvé, il serait certes stérile d'« opposer deux points de vue, l'un national et l'autre européen, sur les droits fondamentaux" puisqu' « il ne saurait y avoir de standards communs qu'enracinés dans des pratiques nationales et, en retour, de protection effective et dynamique sans un contrôle externe, placé entre les mains d'une Cour internationale ».

107 Mais fermeté et cohérence, ensuite, car la garantie européenne des droits fondamentaux n'a de sens que si les droits et libertés conventionnels ne peuvent être impunément foulés aux pieds par les acteurs étatiques. Sauf à renoncer aux impératifs qui ont présidés à la fondation du système européen des droits de l'homme. Et donc à "s'aveugler pour l'avenir $»^{216}$.

108 Quoiqu'il en soit, ces différents atouts européens se rejoignent pour nourrir la résilience juridictionnelle dont la Cour de Strasbourg a fait preuve jusqu'à ce jour. Riche en vertus, cette posture n'est certes pas sans vices. Car la posture du roseau magnifiée par La Fontaine peut aussi être perçue comme un signe de faiblesse ${ }^{217}$. Mais en ces temps troublés, prenons le parti de faire confiance à la Cour européenne pour qu'elle ne plie que rarement. Et surtout, que jamais elle ne rompe.

Rapport annuel 2014 de la Cour européenne des droits de l'homme - Analyse statistique 2014

Conférence de presse annuelle du Président de la Cour européenne des droits de l'homme (Communiqué de presse et Discours du Président Spielmann) - 29 janvier 2015

111 Audience solennelle d'ouverture de l'année judiciaire (Discours du Président Spielmann et du Président Pérez de los cobos Orihuel) et Séminaire "Subsidiarité : Une médaille à deux faces" (Discours du Président Spielmann) 30 janvier 2015

112 Conférence de Bruxelles, 26 et 27 mars 2015, « La mise en œuvre de la Convention européenne des droits de l'homme, notre responsabilité partagée "-Contribution de la Cour et Déclaration commune de 42 ONG de défense des droits de détenus (Communiqué) 
Les Lettres « Actualités Droits-Libertés » (ADL) du CREDOF (pour s'y abonner) sont accessibles sur le site de la Revue des Droits de l'Homme (RevDH) - Contact

\section{NOTES}

1. Mikael Rask Madsen, "The Legitimization Strategies of International Judges: The Case of the European Court of Human Rights ", in iCourts Working Paper Series, $\mathrm{N}^{\circ} 12$, 2014, p. 6: «Importantly, the argument presupposes that temporary crises and controversies are not by definition signs of defeat but instead are indicative of evolution, following, broadly speaking, the ideas of punctuational bursts and punctuated equilibria found in neo-Darwinist theories of evolution. "

2. Discours du Président Spielmann - Audience solennelle, p. 3.

3. Ibid., p. 3. Contra lire notamment notre article « La Cour européenne des droits de l'homme, stratège juridictionnel face aux enjeux brûlants de société", in Revue des droits de l'homme, 8 septembre 2014.

4. v. le Discours du Président Spielmann - Audience solennelle, pp. 3 et 4.

5. Pour reprendre une métaphore déjà employée : ADL du 29 avril 2013.

6. Ibid., p. 1: Le "chiffre astronomique de 160000 requêtes pendantes que nous avions connu en septembre 2011 et qui faisait alors craindre pour la survie du système ».

7. v. ADL du 30 janvier 2013.

8. v. ADL du 12 mai 2014 au point $1_{-}^{\circ}$.

9. 64900 nouvelles requêtes en 2012 (v. ADL du 30 janvier 2013) et 65900 en 2013 (v. ADL du 12 mai 2014 au point $1_{-}^{\circ}$ ); Comp. aux bilans annuels précédents : 64500 requêtes en $2011-\mathrm{v}$. ADL du 29 janvier 2012 ; 61300 en 2010 - v. ADL du 27 janvier 2011 - ; 57100 en 2009 - v. ADL du 29 janvier $2010 ; 40900$ en 2008 - v. ADL du 29 janvier 2009.

10. Rapport annuel 2014, p. 11.

11. Entrée en vigueur du Protocole $n^{\circ} 14$ à la Convention le $1^{\text {er }}$ juin 2010 - ADL du 1er juin 2010 - ; les travaux d'Interlaken en 2010 - ADL du 23 février 2010 - et ceux d'Izmir en 2011 - ADL du 1er mai 2011.

12. Avant-propos du Président Spielmann, in Rapport annuel 2014, p. 5.

13. ADL du janvier 2013 au point $2^{\circ}$.

14. Avant-propos du Président Spielmann, in Rapport annuel 2014, p. 5 ; Les requêtes pendantes devant un juge unique sont passées de 26500 à 8200 au cours de l'année écoulée, soit une réduction spectaculaire de $69 \%$.

15. Rapport annuel 2014, p. 14.

16. Discours du Président Spielmann le 27 juin 2013, p. 7.

17. Le formulaire de requête et les consignes de la Cour.

18. V. le communiqué de presse du 10 janvier 2014.

19. Discours du Président Spielmann, $1219^{\mathrm{e}}$ réunion des Délégués des ministres, 11 février 2015, p. $1:$ «le taux de rejet (des requêtes introduites), en raison du caractère incomplet des documents soumis, s'élève à $23 \%$ ». 
20. Conférence de presse du Président Spielmann, 12'.

21. Rapport annuel 2014, p. 15 : «Plusieurs États ont octroyé des fonds supplémentaires destinés à couvrir le recrutement de juristes par le greffe pour travailler sur l'arriéré des affaires recevables. Le compte spécial créé à cet effet après la Conférence de Brighton a pour l'instant été crédité de plus de 2 millions d'euros par 22 États au total, les contributeurs les plus importants étant la Norvège et l'Allemagne, qui ont versé la moitié de ce montant ».

22. Sur cette question, v. ADL du 30 janvier 2013 au point $1^{\circ}$ et ADL du 27 novembre 2011 au point $2^{\circ}$.

23. Conférence de presse du Président Spielmann, 47' : "Evidemment, dans un monde idéal, si nous avions plus de personnel, plus de moyens financier, on pourrait encore travailler mieux ».

24. Ibid., 50' ; v. aussi Discours du Président Spielmann, $1219^{\mathrm{e}}$ réunion des Délégués des ministres, 11 février 2015, p. 3 : «Il nous faudrait quarante juristes supplémentaires pendant huit ans pour venir à bout de notre arriéré ».

25. v. les discussions en amont et durant la Conférence de Brighton : ADL du 4 mars 2012 et ADL du 23 avril 2012.

26. Discours du Président Spielmann, $1219^{\mathrm{e}}$ réunion des Délégués des ministres, 11 février 2015, p. 2 : «L'ancienneté moyenne du stock continue à s'accentuer puisqu'elle passe de 32,6 mois d'ancienneté en 2013 à 37,6 mois à fin 2014 ».

27. Déclaration de Brighton, $\S 20 \mathrm{~h}$ (v. ADL du 23 avril 2012 au point I) : la Cour droit prendre « la

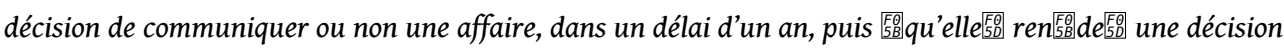
ou un arrêt sur toute affaire communiquée dans un délai de deux ans après sa communication ».

28. Discours du Président Spielmann - Conférence de presse, p. 2 ; En 2013, le Greffier de la Cour, Erik Frigberg, avait indiqué que« 47 \% (de l'arriéré d'affaires) sont des requêtes répétitives 》 (v. ADL du 12 mai 2014 au point $1-B)$.

29. Cf. infra au point 3-B.

30. Pour un riche tour d'horizon des arrêts Rapport annuel 2014, p. 15

31. En 2014, la Grande Chambre a tenu 20 audiences et a rendu 19 arrêts. Au cours de cette même année, 18 affaires ont été renvoyées en Grande Chambre par décision du Collège et 5 l'ont été par dessaisissement d'une formation de Chambre (Rapport annuel 2014, pp. 63-64).

32. ADL du 12 mai 2014 au point 1-B.

33. Pour un tableau complet, v. Rapport annuel 2014, pp. 170 et 171.

34. v. ADL du 12 mai 2014 au point 1-B.

35. Discours du Président Spielmann - Conférence de presse, p. 2 : «L'Italie a fait de très gros efforts au cours de l'année écoulée. En septembre 2014, plus de 17000 affaires étaient pendantes contre l'Italie, ce chiffre a été ramené à 10000 à la fin 2014 »

36. ADL du 12 mai 2014 au point 2-A.

37. A noter que le nombre de requêtes ayant donné lieu au prononcé d'un arrêt s'élève à 2388 en 2014 mais « une proportion importante d'entre elles ont été jointes, ce qui explique que le nombre d'arrêts réellement prononcés soit de 891 » (Analyse statistique 2014, p. 5).

38. Analyse statistique 2014, pp. 4 et 13 : Ce chiffre a "augmenté de $16 \%$ en 2014 (2 198 contre 1890 en 2013). L'augmentation a été de $15 \%$ pour les règlements amiables (1 696 contre 1481 en 2013) et de $23 \%$ pour les déclarations unilatérales (502 contre 409 en 2013) ».

39. Les deux arrêts sur la gestation pour autrui furent les plus retentissants à ce titre : Cour EDH, $5^{\mathrm{e}}$ Sect. 26 juin 2014, Mennesson c. France et Labassée c. France, Resp. Req. n 65192/11 et $\mathrm{n}^{\circ}$ 65941/11 - ADL du 8 septembre 2014.

40. En 2013, deux cas de violation et sept constats de violation conditionnelle furent recensés (ADL du 12 mai 2014 au point 2-A). 
41. Sur la « violation par ricochet », v. la fiche thématique « Expulsions et extraditions ».

42. De 65800 à 56 300, soit une réduction de $3 \%$ (Analyse statistique 2014, p. 5).

43. i.e. la phase d'examen initiale d'une requête par le Greffe aux fins de déterminer s'il y a lieu de l'attribuer à une formation judiciaire.

44. i.e. la phase qui précède la décision de la Cour de communiquer - ou non - la requête au gouvernement de l'Etat défendeur afin d'initier le débat contradictoire.

45. Ce que conteste le Président Spielmann : «Loin de décourager l'introduction de nouvelles requêtes, l'article 47 responsabilise tous les protagonistes par une rigueur accrue servant l'excellence du travail » (Avant-propos du Président Spielmann, in Rapport annuel 2014, p. 5).

46. ADL du 30 janvier 2013 in fine et ADL du 12 mai 2014 au point 2-A.

47. V. la page dédiée à la présentation des requêtes, le guide pratique sur la recevabilité traduit en plusieurs langues, ainsi que les utiles fiches thématiques sur la jurisprudence de la Cour et sur les Etats parties.

48. Pour un exemple, v. Nicolas Hervieu et Serge Slama, «Enfants en rétention : une tragédie franco-européenne en quatre actes ", in CPDH, 6 mars 2012.

49. Analyse statistique 2014, p. 5 : si « la Cour a fait droit à la demande 侮de mesure provisoire硧 dans 216 cas (contre 108 en 2013, soit une augmentation de $100 \%$ ) et l'a rejetée dans 783 cas (contre 818 en 2013, soit une diminution de $4 \%$ ) ", il est précisé que « l'augmentation des demandes accueillies s'explique par 110 requêtes liées au conflit en Ukraine ".

50. Ibid., p. 5 : puisque 216 demandes acceptées et 783 rejetées (pour un total de 999) ont été recensées sur les 1929 introduites en 2014, les 930 autres demandes ont été regardées comme

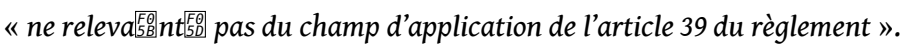

51. V. ainsi l'Avis du CDDH sur les questions à aborder lors de la conférence d'Interlaken, $1^{\text {er }}$ décembre 2009, page 231.

52. Si le non respect des exigences de l'article 47 du Règlement ne peut être corrigé avant l'échéance du délai de six mois.

53. Sur cette critique, v. ADL du 12 mai 2014 au point 2-C.

54. Sur ce point, v. not. Patrice Spinosi, «L'approche d'un praticien français face à la procédure d'examen de la recevabilité des requêtes ", in Quel filtrage des requêtes par la Cour européenne des droits de l'homme?, Pascal Dourneau-Josette et Elisabeth Lambert Abdelgawad (Dir.), Strasbourg, Ed. du Conseil de l'Europe, 2011, p. 253 : Il serait opportun que la Cour s'attache à rendre « la procédure d'examen de la recevabilité des requêtes (...) plus humaine et plus transparente car, plus que d'obtenir satisfaction, les justiciables ont un besoin irréductible d'être écouté ».

55. A ce sujet, v. ADL du 30 janvier 2013 au point $2^{\circ}$ in fine; v. aussi «Entretien avec Dean Spielmann ", in RevDH, 2013, $n^{\circ} 3$.

56. Discours du Président Spielmann, $1219^{\mathrm{e}}$ réunion des Délégués des ministres, 11 février 2015, p. 5 .

57. Ibid., p. 2.

58. Ibid., p. 2 : Dans l'application des exigences de l'article $47 \mathrm{du}$ Règlement, le Président a "demandé que les cas "limites" (lui) soient soumis personnellement» notamment lorsque la particulière vulnérabilité de certains requérants pourrait expliquer le non respect des exigences formelles.

59. En ce sens, v. ADL du 12 mai 2014 au point 1-C in fine: Un dispositif d'alerte et de vigilance, qui associe notamment des acteurs extérieurs au seul Greffe de la Cour ayant traité l'affaire, afin de réexaminer exceptionnellement - et sous l'autorité du Président - certaines décisions d'irrecevabilité qui soulèvent des questions spécifiques et méritent un regard, si ce n'est plus approfondi, à tout le moins collectif.

60. Discours d'ouverture du séminaire du Président Spielmann, 31 janvier 2014, p. 1 (et ADL du 3 septembre 2013. 
61. Denys Simon, «Des influences réciproques entre CJCE et Cour EDH: "Je t'aime, moi non plus ?" ", in Pouvoirs, 2001, n 96, pp. 31-49.

62. CJUE, Ass. Pl. 18 décembre 2014, Avis 2/13.

63. David Szymczak, «L'adhésion de l'Union européenne à la CEDH : Serpent de mer ou Hydre de Lerne?", in Politeïa, 2008, p. 405 ; lire aussi du même auteur, "La perspective d'un contrôle externe des actes de l'Union européenne ", in RDLF, 2014, Chr. 22.

64. Pour un tour d'horizon, v. Xavier Groussot, Tobias Lock et Laurent Pec, « Adhésion de l'Union européenne à la Convention européenne des droits de l'Homme ", in Question d'Europe, 2011, $\mathrm{n}^{\circ} 218$; Tobias Lock, « End of an Epic ? The Draft Agreement on the EU's Accession to the ECHR » (SSRN), in Yearbook of European Law, 2012, pp. 162-197; Paul Gragl, The Accession of the European Union to the ECHR, Hart Publishing, Oxford, 2013, 362 p.; Vicki Kosta, Nikos Skoutaris et Vassilis Tzevlekos (Dir.), The EU Accession to the ECHR, Acte du colloque des 16-17 novembre 2012, Hart Publishing, Oxford, 2014, 402 p. ;

65. Sur ce projet, lire not. ADL du 3 septembre 2013 au point $2^{\circ}$.

66. Art. $218 \S 8 \mathrm{du}$ TFUE.

67. Art. $218 \S 11 \mathrm{du}$ TFUE.

68. Si l'on se limite au seul dispositif de l'avis (\$ 258) : Trois éléments dans le premier paragraphe, un dans le deuxième, deux dans le troisième, et un dans le quatrième et dernier paragraphe.

69. "L'Union adhère à la Convention européenne de sauvegarde des droits de l'Homme et des libertés fondamentales. Cette adhésion ne modifie pas les compétences de l'Union telles qu'elles sont définies dans les traités. »

70. En français, lire Jean-Paul Jacqué, « L'avis 2/13 CJUE. Non à l'adhésion à la Convention européenne des droits de l'homme?", in Droit de l'Union européenne, 23 décembre 2014 ; Henri Labayle, «La guerre des juges n'aura pas lieu. Tant mieux ? ", in GDR-ELSJ, 22 décembre 2014; Roseline Letteron, "Adhésion de l'UE à la Convention européenne des droits de l'homme : La CJUE se lance dans le protectionnisme », in LLC, 21 décembre 2014 ; Marie Gautier, «Ad kalendas graecas ? ", in AJDA, 2015, nº 3 p. 109 ; Fabrice Picod, «La Cour de justice a dit non à l'adhésion de l'Union européenne à la Convention EDH . - Le mieux est l'ennemi du bien, selon les sages du plateau du Kirchberg », in JCP G, n 6, 2015, pp. 145 et s. ; Rostane Mehdi, « Avis 2/13 : le mécanisme de codéfendeur à l'épreuve de l'autonomie du droit de l'Union européenne ", in GDRELSJ, 13 février 2015.

71. En anglais, lire Steve Peers, "The CJEU and the EU's accession to the ECHR: a clear and present danger to human rights protection ", in EU Law Analysis, 18 décembre 2014; Tobias Lock, " Oops! We did it again - the CJEU's Opinion on EU Accession to the ECHR », in Verfassungsblog, 18 décembre 2014; Sionaidh Douglas-Scott, « Opinion 2/13 on EU accession to the ECHR: a Christmas bombshell from the European Court of Justice », in UK Constitutional Law, 24 décembre 2014.

72. Pour une étude approfondie au sein de la Revue des Droits de l'Homme, nous renvoyons à une Lettre ADL à paraître prochainement par Athanase Popov.

73. Henri Labayle, précité : « La posture de la Cour de justice est délibérément politique. Elle ne dissimule en rien la vision qu'elle se fait de la construction l'Union européenne et du droit dont elle est, il est toujours bon de le rappeler, l'interprète authentique en vertu de l'article 19 TUE ».

74. Avis $2 / 13$, $\$ 158$; v. aussi $§ 167$ : «Ces caractéristiques essentielles du droit de l'Union ont donné lieu à un réseau structuré de principes, de règles et de relations juridiques mutuellement interdépendantes liant, réciproquement, l'Union elle-même et ses États membres, ainsi que ceux-ci entre eux, lesquels sont désormais engagés, comme il est rappelé à l'article $1^{\text {er }}$, deuxième alinéa, TUE, dans un " processus créant une union sans cesse plus étroite entre les peuples de l'Europe ». 
75. Ibid. § 174.

76. Marie Gautier, « Ad kalendas graecas? », précité.

77. Fabrice Picod, «La Cour de justice a dit non à l'adhésion de l'Union européenne à la Convention $\mathrm{EDH} »$, précité.

78. Avis $2 / 13$, § 163 :

79. Ibid. $\$ 165$.

80. Ibid. § 156 .

81. Ceci est particulièrement topique pour les deux premières objections de la Cour de justice envers l'accord d'adhésion (v. ainsi § 178-195).

82. Article $1^{\text {er }}$ du protocole $n^{\circ} 8$ relatif à l'adhésion de l'Union à la Convention.

83. v. not. CJCE, 28 mars 1996, Avis 2/94 sur l'adhésion de la Communauté à la convention de sauvegarde des droits de l'homme et des libertés fondamentales.

84. Marie Gautier, «Ad kalendas graecas?", précité.

85. Comp. à cet égard les analyses respectives de Fabrice Picod («La Cour de justice a dit non à l'adhésion de l'Union européenne à la Convention EDH», précité) et de Marie Gautier («Ad kalendas graecas? », précité)

86. Jean-Paul Jacqué, précité.

87. Avant-propos du Président Spielmann, in Rapport annuel 2014, p. 6.

88. Discours du Président Spielmann le 27 juin 2013, p. 5.

89. v. ADL du 3 septembre 2013.

90. Marie Gautier, «Ad kalendas graecas? », précité.

91. Avant-propos du Président Spielmann, in Rapport annuel 2014, p. 6.

92. V. not. Jean-Paul Jacqué, précité : "Que va-t-on dire de cette Union qui porte ses valeurs en bandoulière, mais qui est incapable de se soumettre à un contrôle externe?".

93. Tobias Lock, «Will the empire strike back? Strasbourg's reaction to the CJEU's accession opinion », in Verfassungsblog, 30 janvier 2015.

94. Avant-propos du Président Spielmann, in Rapport annuel 2014, p. 6.

95. En ce sens, lire Tobias Lock, « Will the empire strike back? Strasbourg's reaction to the CJEU's accession opinion ", in Verfassungsblog, 30 janvier 2015;

96. A ce sujet, v. la fiche thématique « Jurisprudence relative à l'Union européenne » et not. Cour EDH, GC, 21 janvier 2011, M.S.S. c. Belgique et Grèce, Req. n 30696/09 - ADL du 21 janvier 2011.

97. Françoise Tulkens, "Oublions la réforme, pensons à l'avenir ", in Cahiers de droit européen, 2012, n², p. 342.

98. v. ADL du 12 mai 2014 au point 2-B.

99. v. notamment à l'occasion de la conférence de Brighton: ADL du 4 mars 2012 et ADL du 23 avril 2012

100. «Protecting human rights in UK », Conservatives, octobre 2014, 8 p.

101. Lire Nicholas Watt et Owen Bowcott, «Tories plan to withdraw UK from European convention on human rights ", in The Guardian, 3 octobre 2014.

102. Lire ADL du 16 octobre 2011 et ADL du 1er novembre 2012 : "A democratic override" évoqué notamment dans le cadre des débats sur le droit de vote des détenus (cf. Michael Pinto-Duschinsky, «Prisoner votes: Strasbourg should give way to national independence ", in The Guardian, 29 octobre 2012 ; lire l'excellente réponse de Fiona de Londras, "Let's not let popularism masquerade as sovereignty on prisoner votes ", in The Guardian, 31 octobre 2012).- ADL du 16 octobre 2011 et ADL du 1er novembre 2012).

103. A ce sujet, lire notamment Jannika Jahn, «The UK's Potential Withdrawal from the European Convention on Human Rights - Just a Flash in the Pan or a Real Threat?» in Verfassungsblog, 17 
décembre 2014; v. aussi David Harris, Michael O'Boyle, Ed Bates et Carla Buckley, «Putting the Potential UK Withdrawal from the ECHR into Perspective », in ECHR Blog, 14 août 2014.

104. Ce que le Gouvernement Cameron avait déjà tenté à l'occasion de la Conférence de Brighton : sur le projet initial de Déclaration du gouvernement britannique, v. ADL du 4 mars 2012.

105. Owen Bowcott, « Conservative retreat from Europe may undermine respect for international law », in The Guardian, 30 septembre 2014; Conor Gearty, « The Tories' proposal for a British bill of rights is incoherent, but they don't care ", in The Guardian, 3 octobre 2014; Editorial du Financial Times, «Britain should stay inside the ECHR », in Financial Times, 17 juillet 2014; Geraldine Van Bueren, « Why I defend the Convention on Human Rights », in The Day, 9 février 2015.

106. Sur les critiques de Dominique Grieve, v. Rajeev Syal, "Cameron warned over 'sabrerattling' with European court of human rights ", in The Guardian, 13 août 2014.

107. Chris Johnston, «Simon Hughes: Tories abandoning human rights to counter Ukip », in The Guardian, 4 octobre 2014.

108. Mikael Rask Madsen, précité, p. 4.

109. David Leigh, «From the archive, 17 November 1980: UK dilemma over European court of human rights ", ", in The Guardian, 17 novembre 2014.

110. V. Philip Leach et Alice Donald, "Hostility to the European Court and the risks of contagion », in UK Human Rights Blog, 21 novembre 2013.

111. « Putin: Russia's withdrawal from ECHR possible », in Rapsi, 14 août 2014.

112. Lire not. Dominique Voegele, «Conseil de l'Europe, et Russie, les histoires d'amour finissent mal...En général », in Europe(s), 21 janvier 2015.

113. Récemment, v. «Ueli Maurer s'attaque à la Convention européenne des droits de l'homme », in RTS info, 21 novembre 2014 ; lire aussi Simon Bradley, « Pourquoi la CEDH est remise en cause en Suisse ", in SWI, 27 novembre 2014.

114. V. ainsi les appels au retrait de Marine Le Pen ou de Guillaume Larrivé et de François Fillon (Paule Gonzales, «La Cour européenne des droits de l'homme dans la ligne de mire de la droite », in Le Figaro, 15 janvier 2015 ; en réponse, lire notre tribune, « Antiterrorisme : la Cour européenne est une alliée » in Libération, 21 janvier 2015).

115. La gestation pour autrui (Cour EDH, $5^{\mathrm{e}}$ Sect. 26 juin 2014, Mennesson c. France et Labassée c. France, Resp. Req. $n^{\circ} 65192 / 11$ et $n^{\circ} 65941 / 11$ - ADL du 8 septembre 2014), les syndicats dans l'armée (Cour EDH, 5 e Sect. 2 octobre 2014, Matelly c. France et ADEFDROMIL c. France, Resp. Req. $\mathrm{n}^{\circ}$ 10609/10 et 32191/09 - Communiqué) ou l'arrestation de pirates (Cour EDH, 5 ${ }^{\mathrm{e}}$ Sect. 4 décembre 2014, Hassan et autres c. France, Req. $\mathrm{n}^{\circ}$ 46695/10 et Ali Samatar et autres c. France, Req. $\mathrm{n}^{\circ}$ 17110/10 - Communiqué).

116. Conférence de presse du Président Spielmann, 24'.

117. Pour preuve, la récente visite du Président Spielmann en Suisse et son discours prononcé le 9 décembre 2014 devant le Parlement se sont soldés par un succès diplomatique (lire Lise Bailat, "Trêve sur les droits humains à Berne ", in 24heures, 9 décembre 2014).

118. A l'aune des arrêts rendus par la Cour en juin 2014, le Conseil d'Etat a ainsi rejeté le recours initié contre la circulaire relative à la délivrance des certificats de nationalité française pour les enfants né par gestation pour autrui : CE, 12 décembre 2014, Association Juristes pour l'enfance et autres, Req. n 367324 - ADL du 2 février 2015.

119. Cour EDH, $5^{\text {e }}$ Sect. 2 octobre 2014, Matelly c. France et ADEFDROMIL c. France, Resp. Req. $\mathrm{n}^{\circ} 10609 / 10$ et $32191 / 09$ - Communiqué.

120. Ainsi, dans son rapport remis au Président de la République le 19 décembre dernier, Bernard Pêcheur a invité les autorités françaises à prendre acte des condamnations européennes et à saisir l'occasion de réformer l'institution militaire en usant de l'importance liberté concédée par la Cour : «Rapport sur le droit d'association professionnelle des militaires », 18 décembre 2014, $110 \mathrm{p}$. 
121. V. ainsi les déclarations de Lord Neuberger, Président de la Cour suprême du Royaume-Uni (Owen Bowcott, «Written UK constitution would limit influence of Strasbourg court, says top judge ", in The Guardian, 13 octobre 2014) et celles de Brenda Hale, sa vice-Président («UK court rulings show move away from European to common law », in The Guardian, 15 août 2014).

122. Pour un exemple frappant, v. ainsi le contentieux relatif au droit de contre-interrogatoire en matière pénale qui a donné lieu le 16 décembre dernier à un arrêt Horncastle $c$. Royaume-Uni (Communiqué), en écho à l'arrêt Al-Khawaja et Tahery c. Royaume-Uni (ADL du 18 décembre 2011).

123. v. ADL du 12 mai 2014 au point 3-C.

124. v. notre article, "Droit de vote des détenus : Histoire sans fin pour un contentieux décisif ", in Rev. trim. dr. h., n 94/2013, p. 456.

125. Nico Krish, «The Backlash against International Courts », in Verfassungsblog, 16 décembre 2014.

126. Ibid.: "Frustrated with the fact that domestic courts have followed Strasbourg in important cases, political actors there seek to create greater distance between the national legal orders and Convention law, thus opening up space for political contestation ".

127. Jean-Claude Mignon et René Rouquet, «La Cour européenne des droits de l'Homme constitue-t-elle une menace pour la démocratie?", in Fondation Robert Schuman, 2 février 2015, Question d'Europe $n^{\circ} 342$

128. En ce sens, lire ADL du 20 octobre 2014 in fine.

129. Pour prendre un exemple récent, il n'est ainsi pas certain que la récente concession de la

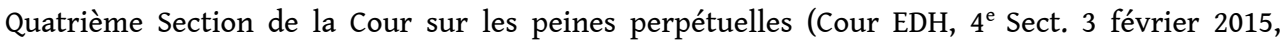
Hutchinson c. Royaume-Uni, Req. $n^{\circ}$ 57592/08 - Communiqué) soit opportune, alors que la Grande Chambre avait été bien plus ferme dans son arrêt 2013 quant à l'encadrement du «droit à l'espoir » (v. ADL du 18 juillet 2013 sur Cour EDH, G.C. 9 juillet 2013, Vinter et autres c. Royaume-Uni, Req. $\mathrm{n}^{\circ}$ 66069/09).

130. v. ADL du 29 janvier 2012.

131. Jean-François Flauss, «Le renouvellement triennal de la Cour européenne des droits de l'homme », in RTDH, 2001, n 47, pp. 712-713.

132. Mikael Rask Madsen, «The Legitimization Strategies of International Judges: The Case of the European Court of Human Rights ", in iCourts Working Paper Series, $\mathrm{N}^{\circ} 12$, 2014, p. 2; Başak Çall, Anne Koch et Nicola Bruch, "The social legitimacy of human rights courts: A grounded interpretivist theory of the legitimacy of the European Court of Human Rights ", in Human Rights Quarterly, vol. 35, 2013, pp. 955-984. V. aussi la Déclaration de Brighton : «l'autorité et la crédibilité de la Cour dépendent en grande partie de la qualité de ses juges et des arrêts qu'ils rendent » (\$21).

133. A ce sujet, v. l'illustre chronique de Jean-François Flauss à la Revue Trimestrielle des Droits de l'Homme: en 1998, $\mathrm{n}^{\circ} 35$, pp. 435-464; en 2001, $\mathrm{n}^{\circ} 47$, pp.693-713; en 2005, $\mathrm{n}^{\circ} 61$, pp. 5-32; puis en $2008, \mathrm{n}^{\circ} 75$, pp. 713-741.

134. Laquelle est déterminante car, ainsi que l'a souligné la Déclaration de Brighton, «le haut niveau des juges élus à la Cour est fonction de la qualité des candidats présentés à l'Assemblée parlementaire. Le choix de candidats ayant la plus haute envergure possible, opéré par les États parties, est de ce fait primordial pour préserver le succès de la Cour » (\$21).

135. Sur la procédure de désignation, lire Commission des questions juridiques et des droits de l'homme, "Procédure d'élection des juges à la Cour européenne des droits de l'homme », 26 septembre 2014, AS/Jur/Inf (2014) 03 rév 5 ; lire aussi Laurence Burgorgue-Larsen, « Des idéaux à la réalité. Réflexions comparées sur les processus de sélection et de nomination des membres des Cours européenne et interaméricaine des droits de l'homme ", in Revue des Droits de l'Homme, $2014, \mathrm{n}^{\circ} 6,26 \mathrm{p}$.

136. Lire ADL du 1er mai 2012. 
137. En juin 2013 et novembre 2014 (v. le tableau de suivi concernant les « Prochaines élections des juges à la Cour européenne des droits de l'homme »).

138. Laurence Burgorgue-Larsen, précité.

139. Son mandat s'achevait normalement le 30 avril 2017 mais elle a démissionné avec prise d'effet au 1er mars 2015.

140. Son mandat s'est achevé le 3 avril 2014, mais il est resté en fonction de façon temporaire. Et ce, manifestement en raison du retard pris par la Serbie pour soumettre à l'Assemblée parlementaire une liste de trois candidats (attendue le 12 novembre 2013, cette liste n'a été présente que le 2 décembre 2014).

141. Sur cet enjeu et plus précisément sur la "représentation du sexe sous-représenté » parmi les juges de la Cour, v. ADL du 1er mai 2012.

142. Pour un témoignage notamment à ce sujet, lire "Entretien avec Françoise Tulkens ", in RevDH, 2013, $\mathrm{n}^{\circ}$ 3. L'ancienne juge et vice-Présidente de la Cour y souligne notamment qu'en tant que juges, "nous arrivons tous à Cour avec des expériences et aussi des tempéraments personnels différents".

143. Mikael Rask Madsen, «The Legitimization Strategies of International Judges: The Case of the European Court of Human Rights ", in iCourts Working Paper Series, $N^{\circ} 12,2014$, p. 22-23.

144. Ibid. p. 25.

145. Sur le défi diplomatique, v. not. ADL du 12 mai 2014 au point 2.

146. Sur les différentes élections au cours de l'année 2012, v. ADL du 1er mai 2012;ADL du 28 juin 2012 et ADL du 10 septembre 2012.

147. V. la liste des Présidents.

148. Pour reprendre une expression plus finement tracée par l'intéressé lui-même: "Si le président de la Cour est "primus inter pares", il l'est à l'instar d'un "chef d'orchestre", sans pour autant se retrouver au pupitre du premier violon. » (« Entretien avec Dean Spielmann », in RevDH, 2013, n³).

149. Pour reprendre les mots de l'intéressé lui-même: Discours du Président Spielmann Audience solennelle, p. 1.

150. ADL du 29 janvier 2012.

151. ADL du 10 septembre 2012

152. Article 25 de la Convention : "La Cour réunie en Assemblée plénière (...) a) élit, pour une durée de trois ans, son président et un ou deux vice-présidents; ils sont rééligibles; (...); c) élit les présidents des chambres de la Cour, qui sont rééligibles; »

153. En novembre prochain.

154. Durant ce mois de février (v. le séminaire organisé en son honneur le 13 février).

155. Article 25 de la Convention : «La Cour réunie en Assemblée plénière 嶇...區 e) élit le greffier et un ou plusieurs greffiers adjoints » (v. l'organigramme du Greffe).

156. Mikael Rask Madsen, précité, p. 5: « The question is how they manage to remain powerful in what often appears to be hostile environments. Or, more specifically: what explains the successes and failures among the various international courts established since the beginning of the twentieth century in terms of legitimacy? What are their legitimization strategies? »

157. V. ainsi Nico Krish, "The Backlash against International Courts », in Verfassungsblog, 16 décembre 2014.

158. Cf. supra au point 2-B.

159. Sur le Royaume-Uni, v. Owen Bowcott, « UK broke law in fewer than $1 \%$ of European human rights cases in 2014 ", in The Guardian, 29 janvier 2015 ; Sur la Russie, v. les résultats statistiques pour l'année 2014 qui révèlent une nette embellie : supra au point 1-A.

160. Pour un exemple paroxystique d'une telle situation, v. le contentieux du droit de vote des détenus au Royaume-Uni : ADL du 1er novembre 2012 au point $2^{\circ}$ et notre article, « Droit de vote 
des détenus: Histoire sans fin pour un contentieux décisif ", in Rev. trim. dr. h., n 94/2013, pp. 433-456; v. aussi ADL du 18 juillet 2013 in fine.

161. V. notamment la Déclaration d'Interlaken en 2010 (§ 7), celle d'Izmir en 2011 (point E) ou encore celle de Brighton en 2012 (§ 20.c).

162. v. not. le rapport du Comité directeur pour les droits de l'homme (CDDH) contenant «des conclusions et éventuelles propositions de mesures sur les moyens de régler le grand nombre de requêtes résultant de problèmes systémiques identifiés par la Cour » (7 juin 2013) et la réponse de la Cour à ce rapport (20 octobre 2014 ;

163. Discours du Président Spielmann - Conférence de presse, p. 2.

164. Cf. supra au point 1-A.

165. Discours du Président Spielmann, 204ème Réunion des Délégués des Ministres, 2 juillet 2014, p. 2 : " J'ai la conviction que le modèle que nous avons utilisé, mis en œuvre par la section de filtrage, n'a pas épuisé toutes ses potentialités. Cette section de filtrage, très opérationnelle, et qui a désormais moins d'affaires de juge unique, va maintenant s'attaquer aux affaires répétitives et ce, en suivant les méthodes expérimentées avec succès pour les affaires de juge unique, ce que nous appelons le "one in, one out". ».

166. Conférence de presse, 43 '.

167. Discours du Président Spielmann - Conférence de presse, p. 3.

168. v. ADL du 23 avril 2012.

169. En ce sens, Conférence de presse du Président Spielmann, 49' : Avec la conférence de Bruxelles, et «à juste titre, on se focalisera non plus sur la Cour mais sur les Etats"; v. aussi le rapport de M. Pozzo di Borgo, «L'efficacité de la Convention européenne des droits de l'homme : la Déclaration de Brighton et au-delà », 10 décembre 2014, AS/ $\operatorname{Jur}(2014) 33$.

170. Evoqué dans le Discours du Président Spielmann, $1219^{\mathrm{e}}$ réunion des Délégués des ministres, 11 février 2015, p. 5.

171. Ibid.

172. A ce sujet, v. le séminaire dédié à la subsidiarité qui a précédé l'audience solennelle.

173. Discours de Francisco Pérez de los Cobos Orihuel, Président du Tribunal constitutionnel espagnol

174. Dean Spielmann, "Whither the Margin of Appreciation?», in UCL - Current Legal Problems (CLP) lecture, 20 mars 2014, pp. 11-12.

175. Robert Spano, "Universality or Diversity of Human Rights? Strasbourg in the Age of Subsidiarity », in Human Rights Law Review, 2014, vol. 14, n 3, pp. 487-502.

176. En ce sens, lire ADM de mai 2014

177. Contribution de la Cour à la Conférence de Bruxelles, 25 janvier 2015, § 4.

178. Ibid. §5.

179. Jennifer Marchand, «Prévention et dissuasion dans la jurisprudence de la Cour européenne des droits de l'homme ", in RFDA, nº 6, 2014, pp. 1149-1157.

180. v. ADL du 11 février 2013 sur Cour EDH, G.C., 7 février 2013, Fabris c. France, Req. n 16574/08, $\S 75$ : «L'obligation de prévenir, avec diligence, de nouvelles violations semblables à celles constatées dans les arrêts de la Cour [qui pèse sur l'Etat] entraîne l'obligation pour le juge national d'assurer [...] le plein effet des normes de la Convention, telles qu'interprétées par la Cour ».

181. Déclaration commune d'organisations actives dans la défense en justice des droits des détenus dans la perspective de la Conférence de Bruxelles, § 21.

182. Lire Serge Slama, "Une décision de la Cour EDH condamnant la France constitue un changement de circonstances de droit permettant de surmonter un brevet de constitutionnalité », in RevDH, 29 août 2014.

183. Crim. 17 décembre 2014, $n^{\circ}$ 14-90.043.

184. v. not. Jennifer Marchand, précité. 
185. Conférence d'Oslo des 7 et 8 avril 2014 sur l'avenir à long terme de la Cour européenne des droits de l'homme, organisé par PluriCourts sous les auspices du Conseil de l'Europe - le programme.

186. Observations finales de M. Philippe Boillat, p. 2.

187. Conférence de presse du Président Spielmann, 49'.

188. Cour EDH, $4{ }^{\text {e }}$ Sect. 10 février 2015, McHugh et autres c. Royaume-Uni, Req. nº 51987/08 Communiqué.

189. Ibid., § 11 .

190. v. Carl Gardner, « Britain's got it way its way on prisoners' votes - so why withdraw from the ECHR? ", in Head of Legal, 11 février 2015; Pour la réaction d'un détenu à cette décision, lire aussi Carmen Fishwick, «UK prisoners banned from voting: 'Can you put a value on the loss of this right?' ", in The Guardian, 12 février 2015.

191. v. en particulier les travaux du Comité d'experts sur la réforme de la Cour et en particulier les derniers travaux du Groupe de rédaction " $F$ " réalisés au cours de la réunion des 19 au 21 mars 2014 et ceux à venir les 14 et 16 mai prochains; v. aussi le rapport «Assurer la viabilité de la Cour de Strasbourg: les insuffisances structurelles dans les Etats Parties » (2013) ainsi que le rapport de l'ONG Open Justice Society Initiative (2012).

192. Déclaration commune d'organisations actives dans la défense en justice des droits des détenus, $\$ 10$.

193. ADL du 12 mai 2014 au point 3-A.

194. Art. 46 Al. 2 de la Convention : "L'arrêt définitif de la Cour est transmis au Comité des Ministres qui en surveille l'exécution ».

195. Contribution de la Cour à la Conférence de Bruxelles, 25 janvier 2015, § 13.

196. Pour un tour d'horizon approfondie de la question, lire Katarzyna BlayGrabarczyk, Mustapha Afroukh et Aurélia Schahmaneche, "Le contrôle de l'exécution des arrêts de la Cour européenne des droits de l'homme ", in RFDA, n 5, 2014 pp. 935-945

197. ADL du 12 mai 2014 au point 3-A.

198. V. ADL du 26 mars 2011 et la fiche thématique « Arrêts pilotes » : l'Etat condamné se voit signifié les différentes mesures de redressement qu'il doit adopter pour faire cesser une violation systémique de la Convention.

199. Contribution de la Cour à la Conférence de Bruxelles, 25 janvier 2015, § 14.

200. Ibid.: "On pourrait envisager que, dans certains types d'affaires, les parties soient invitées à formuler des observations sur l'efficacité de l'identification de mesures de redressement spécifiques dans l'arrêt de la Cour. Dans des cas exceptionnels, cela pourrait même faire l'objet d'une phase distincte de la procédure devant la Cour, qui viendrait après l'arrêt au principal, selon des modalités comparables à la pratique actuelle et occasionnelle de réserver l'application de l'article 41 ».

201. V. ADL du 12 mai 2014 au point 3-A.

202. Contribution de la Cour à la Conférence de Bruxelles, 25 janvier 2015, § 15.

203. Cour EDH, G.C., 30 juin 2009, Verein Gegen Tierfabriken Schweiz (VGT) c. Suisse ( $\left.n^{\circ} 2\right)$, Req. $\mathrm{n}^{\circ}$ 32772/02, 90 ; «la réouverture d'une procédure ayant violé la Convention n'est pas une fin en soi, elle n'est qu'un moyen - certes privilégié - susceptible d'être mis en cuvre en vue d'un objectif: l'exécution correcte et entière des arrêts de la Cour "

204. v. not. Cour EDH, Dec. $2^{\text {e }}$ Sect. 2 juillet 2013, Hulki Günes c. Turquie, Req. $n^{\circ} 17210 / 09, \S 54-$ Communiqué. 
205. Contribution de la Cour à la Conférence de Bruxelles, 25 janvier 2015, § 15 : «Pour ce qui concerne les affaires pénales et administratives, la réouverture des procédures est susceptible d'offrir au requérant la réparation la plus complète pour la violation de ses droits fondamentaux en le rétablissant dans ses droits autant que faire se peut ».

206. Cour EDH, G.C. 5 février 2015, Bochan c. Ukraine ( $n^{\circ}$ 2), Req. $n^{\circ}$ 22251/08 - Communiqué.

207. Elisabeth Lambert-Abdelgawad, «L'exécution des décisions de justice européenne », in AFDI, 2006, $\mathrm{n}^{\circ} 52$, p. 679 (cité par Katarzyna Blay-Grabarczyk, Mustapha Afroukh et Aurélia Schahmaneche, précité).

208. «Entretien de Françoise Tulkens », in RevDH, n³, juin 2013

209. V. ADL du 30 janvier 2013 in fine.

210. Discours de la juge Laffranque lors du séminaire «La mise en œuvre de la Convention en période de crise économique », 25 janvier 2013.

211. V. notamment le parlementaire britannique David Davies («Paris attacks show need to scrap Human Rights Act », 8 janvier 2015) ou les députés français Guillaume Larrivé, François Fillon et Valérie Pécresse ( La Cour européenne des droits de l'homme dans la ligne de mire de la droite ", in Le Figaro, 15 janvier 2015).

212. "Antiterrorisme : la Cour européenne est une alliée " in Libération, 21 janvier 2015 ; N.B. : Nous remercions le Président Spielmann d'avoir évoqué ces quelques mots issus de notre tribune dans son discours d'audience solennelle.

213. Dean Spielmann, «The European Court of Human Rights as guarantor of a peaceful public order in Europe », Discours au Gray's Inn, 12 novembre 2014.

214. Mikael Rask Madsen, «The Legitimization Strategies of International Judges: The Case of the European Court of Human Rights », in iCourts Working Paper Series, $N^{\circ} 12,2014$, p. 11.

215. v. ADL du 12 mai 2014 au point 3-A

216. Pour reprendre les mots de Président allemand Richard von Weizsäcker - récemment décédé - prononcés le 8 mai 1985 dans son discours du $40^{\mathrm{e}}$ anniversaire de la capitulation allemande: "Celui qui ferme les yeux devant le passé s'aveugle pour l'avenir. Celui qui ne veut pas se rappeler l'inhumain s'expose aux nouveaux risques d'infection ».

217. v. "Droit de vote des détenus : Histoire sans fin pour un contentieux décisif ", in Rev. trim. dr. h., nº 94/2013, pp. 446 et s.

\section{RÉSUMÉS}

Tenir la digue des droits fondamentaux en Europe face aux bourrasques étatiques, aux flots de requêtes et aux tempêtes sécuritaires. A l'évidence, et plus que jamais, la mission de la Cour européenne des droits de l'homme n'est guère aisée. Mais le bilan de l'année écoulée conforte indéniablement la juridiction strasbourgeoise, ne serait-ce qu'avec la poursuite du spectaculaire désengorgement de son prétoire. Certes, le regrettable coup d'arrêt porté au processus d'adhésion de l'Union européenne à la Convention assombrit quelque peu l'horizon du système européen des droits de l'homme. Certes également, les défis restent nombreux pour une juridiction dont l'autorité est constamment mise à l'épreuve et dont l'avenir sera de nouveau discuté lors d'une conférence internationale à Bruxelles en mars prochain. Au surplus, une page va se tourner en 2015, avec la fin du mandat du Président Dean Spielmann et de celui de plus dix autres juges 
sur les quarante-sept que compte la Cour. Mais un nouveau chapitre de l'histoire européenne s'ouvrira alors. Et nul doute qu'il s'écrira lui aussi à l'encre de la résilience juridictionnelle.

\section{AUTEUR}

\section{NICOLAS HERVIEU}

CREDOF - Université de Paris-Ouest Nanterre 